\title{
The Horizontal Spectrum of Vertical Velocities near the Tropopause from Global to Gravity Wave Scales 0
}

\author{
ULRICH SCHUMANN \\ Deutsches Zentrum für Luft- und Raumfahrt, Institut für Physik der Atmosphäre, Oberpfaffenhofen, Germany
}

(Manuscript received 8 June 2019, in final form 11 September 2019)

\begin{abstract}
Vertical motions are fundamental for atmospheric dynamics. Compared to horizontal motions, the horizontal spectrum of vertical velocity $w$ is less well known. Here, $w$ spectra are related to spectra of horizontal motions in the free atmosphere near the tropopause from global to gravity wave scales. At large scales, $w$ is related to vertically averaged horizontal divergent motions by continuity. At small scales, the velocity energy spectra reach anisotropy as in stably stratified turbulence. Combining these limits approximates the $w$ spectrum from global to small scales. The $w$ spectrum is flat at large scales when the divergent spectrum shows a -2 slope, reaches a maximum at mesoscales after transition to $-5 / 3$ slopes, and then approaches a fraction of horizontal kinetic energy. The ratio of vertical kinetic energy to potential energy increases quadratically with wavenumber at large scales. It exceeds unity at small scales in stratified turbulence. Global and regional simulations and two recent aircraft measurement field campaigns support these relationships within 30\% deviations. Energy exchange between horizontal and vertical motions may contribute to slope changes in the spectra. The model allows for checking measurement validity. Isotropy at large and small scales varies between the datasets. The fraction of divergent energy is $40 \%-70 \%$ in the measurements, with higher values in the stratosphere. Spectra above the tropopause are often steeper over mountains than over oceans, partly with two $-5 / 3$ subranges. A total of $80 \%$ of $w$ variance near the tropopause occurs at scales between about 0.5 and $80 \mathrm{~km}$.
\end{abstract}

\section{Introduction}

Horizontal wind and temperature measurements from long-distance commercial aircraft flights at midlatitudes in the upper troposphere and lower stratosphere show a characteristic spectrum of atmosphere dynamics (Nastrom and Gage 1985; Lindborg 1999). Not all measurements show the same characteristics (Gao and Meriwether 1998; Cho et al. 1999), but global highresolution atmosphere models show similar canonical spectra (Hamilton et al. 2008; Burgess et al. 2013; Skamarock et al. 2014; Malardel and Wedi 2016). The large-scale geostrophic motions are interpreted as quasi two-dimensional (2D) with kinetic and potential energy spectra exhibiting spectral steepness near -3 while the mesoscale motions often show a flatter spectrum, with slopes near $-5 / 3$ (Gage and Nastrom 1986). The slope

\footnotetext{
Supplemental information related to this paper is available at the Journals Online website: https://doi.org/10.1175/JAS-D-190160.s1.

Corresponding author: Ulrich Schumann, ulrich.schumann@dlr.de
}

transition scale $L$ occurs between about 300 and $800 \mathrm{~km}$ wavelengths, differing in the stratosphere and troposphere (Bierdel et al. 2016). The quasigeostrophic motions at scales $\lambda>L$ are mainly rotational with low horizontal divergence (Charney 1971; Gill 1982). Mesoscales $(\lambda<L)$ have about equal shares of rotational and divergent horizontal motions (Blažica et al. 2013; Lindborg 2015). Finally, turbulence at small scales is near isotropic and mainly rotational (Tennekes and Lumley 1972). The canonical spectrum is relevant, for example, for numerical weather predictability, which is lower for a flatter spectrum because of shorter turnover time scales (Lorenz 1969). Considerable progress has been made in the understanding of these spectra, suggesting multiple reasons for specific spectral shapes (Li and Lindborg 2018; Selz et al. 2019).

The horizontal spectrum of vertical velocity has been discussed relatively little (Craig and Selz 2018), mainly because of high variability, missing universal theory, and only few measurements of $w$ spectra covering the mesoscales (Bacmeister et al. 1996; Gao and Meriwether 1998; Callies et al. 2016). Vertical wind drives conversion between potential and kinetic energy, gravity 
waves, and turbulence at increasingly smaller scales (Lorenz 1960; Fritts and Alexander 2003), fundamental for atmospheric dynamics. Together with the spectrum of horizontal motions, the $w$ spectrum describes the atmospheric flow energetics as a function of spatial scales (Skamarock et al. 2019), determines the required resolution in numerical models (Frehlich and Sharman 2008), and the amount of subgrid-scale contributions (Lilly 1967). The spectrum may influence cloud microphysics (Barahona et al. 2017; Kärcher et al. 2019) and horizontal cloud scales. Frequency spectra [e.g., from radar (Li et al. 2018) and balloons (Podglajen et al. 2016)] may be comparable to wavenumber spectra when Taylor's hypothesis of frozen motions applies (Bacmeister et al. 1996; Craig and Selz 2018). Vertical wind at large horizontal scales is mostly weak and difficult to determine (Holton 2012; Bony and Stevens 2019). Models and observations often show a maximum of $w$ variance at the smallest resolved scales, leaving the ultimate explanation of this maximum open (Sharman et al. 2012; Zentek et al. 2016; Smith and Kruse 2017).

The atmosphere in the upper troposphere and lower stratosphere is often strongly stratified, with maximum stratification in the tropopause inversion layer (Birner et al. 2002; Gisinger et al. 2017). Stratified turbulence exhibits vertical velocities often far smaller than horizontal ones and layered horizontal motions with strong vertically rotational horizontal components (Lilly 1983). Horizontal and vertical kinetic and potential energies of linear inertial gravity waves are connected by dispersion laws at frequencies between the Coriolis and the BruntVäisälä frequency (Gill 1982). Strong vertical winds are observed regionally in nonlinear gravity waves and turbulence, for example, over mountains and convection (Sharman et al. 2012). Mountain waves can be simulated numerically in agreement with observations (Smith and Kruse 2017). For small-scale turbulence, at scales smaller than the Ozmidov scale (Riley and Lindborg 2008), the spectrum of vertical and horizontal velocities is linked by isotropy and mass continuity (Tennekes and Lumley 1972).

Recently, wind and temperature have been measured in long-distance flights with well-instrumented jet aircraft operating in the lower stratosphere and upper troposphere during field experiments over the South Pacific near New Zealand (Fritts et al. 2016) and over the North Atlantic near Iceland (Schäfler et al. 2018). Accurate wind measurements are demanding in particular for fast-flying jets because the wind vector is the relatively small difference between the measured velocity vector of the aircraft with respect to Earth and the measured velocity vector of the air with respect to the aircraft (Lenschow 1986). The measurements combine position and attitude data from inertial navigation platforms and satellite navigation systems, and air velocity and angleof-attack data from static and dynamic pressure and temperature sensors at the aircraft fuselage and nose (Cooper et al. 2014; Mallaun et al. 2015; Giez et al. 2017). These techniques allow to derive spectra of vertical energy and momentum fluxes in mountain gravity waves (Smith et al. 2016). Occasionally, $w$ spectra were observed for selected flight legs showing peaks at wavelengths of order 4-12 km, which are partially explainable with mesoscale models (Smith and Kruse 2017). Aircraft typically do not fly steady and straight, but undergo ridged body oscillations at frequencies between 0.01 and $0.1 \mathrm{~Hz}$. The vertical body velocity is often larger than vertical air velocity, depending on aircraft design, speed, and autopilot control (Nelson 1998). The aircraft oscillations may be interfering with wind measurements at $4-10 \mathrm{~km}$ wavelengths. Aircraft measurement disturbances and vertical motions at constant pressure levels were noted before, but regarded as unimportant for horizontal velocity spectra at large wavelengths (Nastrom and Gage 1985; Gao and Meriwether 1998; Frehlich and Sharman 2010). As we will show, some $w$ spectra show maxima ("peaks") at horizontal scales corresponding to flight height, in particular near the tropopause over mountains. A wide maximum in $w$ spectra at about 10 $20 \mathrm{~km}$ wavelengths was observed also in airborne Doppler lidar observations of tropospheric mountain waves (Witschas et al. 2017). In other cases, in particular over oceans, the measured $w$ spectra are flatter without such a maximum (Gao and Meriwether 1998).

In this paper we suggest a hypothesis that connects the horizontal spectrum of vertical kinetic energy with horizontal spectra of horizontal kinetic and potential energy and discuss possible implications. Published model results (Skamarock et al. 2014; Selz et al. 2019) and an analysis of two recent airborne field campaigns (Fritts et al. 2016; Schäfler et al. 2018) are used to discuss our understanding of the $w$ spectra from models and related measurements.

\section{The hypothesis}

Vertical wind $w$ is obviously connected to horizontal wind components $u$ and $v$ by mass continuity, which for an incompressible fluid in Cartesian coordinates $(x, y, z)$ implies,

$$
\frac{\partial w}{\partial z}=-\left(\frac{\partial u}{\partial x}+\frac{\partial v}{\partial y}\right)
$$

Integrating Eq. (1) over height from the ground $(z=0)$ to a height $z=h$ shows that $w(h)$ is related to horizontal winds kinematically (Holton 2012): 


$$
w(h)-w(0)=-\int_{0}^{h}\left(\frac{\partial u}{\partial x}+\frac{\partial v}{\partial y}\right)=-h\left(\frac{\partial \bar{u}}{\partial x}+\frac{\partial \bar{v}}{\partial y}\right) .
$$

Here, the bars in $\bar{u}$ and $\bar{v}$ denote the vertical average. We start with the simple case of zero surface height and $w(0)=0$. The vertical wind $w$ at ground drops from the mass budget for vertically impermeable rigid ground, but Eq. (2) would require the height $h$ within the differentials for variable surface height. Also density variations from adiabatic pressure changes and from internal heat sources are generally important (Egger and Hoinka 2019). We leave variable density and orography for later discussion. As noted by Margules in 1904 (Lynch 2003), vertical wind derived from the continuity equation is very error prone at large scales (Holton 2012). The Helmholtz decomposition (Wippermann 1957; Lorenz 1960; Koshyk and Hamilton 2001) is therefore essential for this approach. It allows splitting the horizontal wind vector into a rotational and a divergent part. By definition, only the divergent part contributes to the vertical wind. Equation (2) implies that Fourier modes $\hat{w}$ in $w=\sum \hat{w} \exp (i \mathbf{k x})$ are related to Fourier modes of $\bar{u}$ and $\bar{v}$ with derivatives in the divergence term transforming into multiplications with the corresponding wavenumber components. Hence, the kinetic energy of $w(h)$ in wavenumber space is related to the sum of the energies of $\bar{u}$ and $\bar{v}$ :

$$
\hat{w} \hat{w}^{*}=h^{2}\left[k_{x}^{2} \widehat{\bar{u}} \widehat{\bar{u}}^{*}+k_{x} k_{y}\left(\widehat{\bar{u}} \widehat{\bar{v}}^{*}+\widehat{\bar{v}} \widehat{\bar{u}}^{*}\right)+k_{y}^{2} \widehat{\bar{v}} \widehat{\bar{v}}^{*}\right],
$$

with asterisks indicating complex conjugate values. Because of missing information on the scaling of anisotropy (Nastrom et al. 1997; Bühler et al. 2017), we assume horizontal isotropy (vertical axisymmetry) in which the spectra depend only on the wavenumber magnitude $k=\left(k_{x}^{2}+k_{y}^{2}\right)^{1 / 2}$ with zero mean correlations between $\widehat{\bar{u}}$ and $\widehat{\bar{v}}$. Let $E_{w}$ and $E_{D}$ be the ensemble-mean (denoted by angle brackets) spectral energy densities per wavenumber interval $d k$ of $\left\langle\hat{w} \hat{w}^{*}\right\rangle / 2$ and of the vertically averaged divergent horizontal velocities $\left\langle\widehat{\bar{u}} \widehat{\bar{u}}^{*}+\widehat{\bar{v}} \widehat{\bar{v}}^{*}\right\rangle / 2$ integrated over rings $2 \pi k d k$ at given $h$. Because of Eq. (3), these spectra are related as

$$
E_{w}(k, h)=h^{2} k^{2} E_{D}(k, h) .
$$

This relationship should be exact in a horizontally isotropic atmosphere with constant density at height $h$ over a flat surface. To allow for applications to measured or modeled spectra of divergent kinetic energy $E_{d}(k, h)$ at given height $h$, we relate $E_{D}(k, h)$ to $E_{d}(k, h)$ at this height. The horizontal spectra $E_{D}$ of vertically averaged velocities and $E_{d}$ of velocities at a fixed height differ in magnitude. Their ratio may be expressed by a filter function:

$$
E_{D}(k, h)=F(h k) E_{d}(k, h) .
$$

Here, we suggest an approximate filter function:

$$
F(k h)=\frac{1}{\beta^{-2}+\alpha^{-1}(h k)^{2}},
$$

with two yet open parameters $\alpha$ and $\beta$. Inserting Eqs. (5) and (6) into Eq. (4) results into

$$
E_{w}(k, h)=\frac{\alpha\left(h_{\mathrm{eff}} k\right)^{2}}{\alpha+\left(h_{\mathrm{eff}} k\right)^{2}} E_{d}(k, h) .
$$

The filter function is constructed such that Eq. (7) simplifies for very small and very large wavenumbers. In particular,

$$
E_{w}(k, h)=\left(h_{\mathrm{eff}} k\right)^{2} E_{d}(k, h), \quad h k \ll 1
$$

at small wavenumbers, independent of $\alpha$. In the opposite limit,

$$
E_{w}(k, h)=\alpha E_{d}(k, h)=\alpha d E_{h}(k, h) ; \quad h k \gg 1,
$$

independent of $\beta$. Here we have introduced $E_{d}=d E_{h}$, where $E_{h}(k, h)$ is the measurable spectrum of horizontal kinetic energy ( $u$ and $v$ ) and $d$ an estimated fraction of divergent motions in this spectrum.

The physics behind this filter function is that mass continuity controls the relationship between $E_{w}$ and $E_{D}$, Eq. (4). For a vertically uniform flow in a layer of depth $h$ with long-wave motions, $E_{D} \approx E_{d}$. For vertically variable long-wave motions, $E_{D}$ differs from $E_{d}$ by the factor $\beta^{2}$. The factor will be the smaller the more variable the horizontal divergent layered motions. Here, the effective depth controlling $w$ is $h_{\text {eff }}=\beta h$; $h_{\text {eff }}$ measures the depth of layers with effectively uniform divergent flow. At large heights and large wavenumbers, $E_{w}$ is no longer controlled by mass continuity from the layer mean divergence but more by local dynamics. We assume that this dynamics, in the ensemble average over many realizations, tends to approach near isotropy, so that $E_{w}$ approaches $\alpha E_{d}$ up to a remaining anisotropy of factor $\alpha$, but without the factor $(h k)^{2}$. These properties are approximated by the filter function $F$. The ratio $d$ varies with $k$ and $h$ and with the flow dynamics because divergent and rotational motions follow different conservation laws (Wippermann 1957; Tennekes and Lumley 1972). The value of $d$ is small for low wavenumbers and is variable and on the order of 0.5 at mesoscales (Li and Lindborg 2018).

Subsequently we relate $\alpha$ and $\beta$ to known properties of the velocity field. The ensemble mean kinetic energy $E_{D}$ of vertically averaged horizontal wind (e.g., from $\bar{u}$ ), 
is related to the vertical correlations between winds at altitudes $z^{\prime}$ and $z^{\prime \prime}$ by

$$
\left\langle\bar{u}^{2}\right\rangle=\left\langle\left[\frac{1}{h} \int_{0}^{h} u(z) d z\right]^{2}\right\rangle=\frac{1}{h^{2}} \int_{0}^{h} \int_{0}^{h}\left\langle u\left(z^{\prime}\right) u\left(z^{\prime \prime}\right)\right\rangle d z^{\prime} d z^{\prime \prime},
$$

and similarly for $\bar{v}$. At large scales, $E_{D} / E_{d} \approx \beta^{2}$, see Eqs. (5) and (6). Hence, $\beta^{2}$ could be computed for known vertical profiles of horizontal wind or for given ensemble-mean correlations. For example, $\beta=1 / 2$ for a wind profile increasing linearly from 0 at ground. For vertically more variable divergent wind fields, $\beta$ can be considerably smaller. The correlations are the larger the steeper the spectra of horizontal velocity fluctuations decay with vertical wavenumber, causing large $\beta$ for geostrophic motions with -3 spectra in both the horizontal and vertical wavenumbers (Charney 1971).

The values of $\alpha=E_{w} / E_{d}$ and $\alpha d=E_{w} / E_{h}$ depend on the high wavenumber spectra; see Eq. (9). For given $E_{h}, E_{w}$ depends on the product $\alpha d$; see Eq. (9). In incompressible fully turbulent inertial-subrange turbulence (Kolmogorov 1941), $E_{w} / E_{h}$ approaches a fixed value of 4/7 (Hinze 1959). For linear hydrostatic gravity waves in uniformly stratified atmospheres for high intrinsic frequencies near the Brunt-Väisälä frequency, $E_{w} / E_{h}$ approaches one; see the appendix. However, in the atmosphere isotropic turbulence and linear gravity waves occur only occasionally. Instead, the free atmosphere is mostly stably stratified with occasional turbulent patches and $E_{w} / E_{h}<1$ (Riley and Lindborg 2008).

From a fit of the model to the data from all individual flights presented below, for fixed $d=1 / 2$, we find mean values and standard deviations of $\alpha=0.5 \pm 0.2$ and $\beta=$ $0.11 \pm 0.09$. From comparisons to global and regional models we find that $\beta$ varies with height. Our hypothesis is that Eq. (7), and the concept behind it, approximate the relationship between vertical and divergent horizontal motions, and that $\alpha=0.5$ and $\beta$ between 0.05 and 0.5 provides useful approximations for a range of applications.

The hypothetical spectral relationship is illustrated in Fig. 1, which shows schematically horizontal energy spectra for divergent and total (divergent plus rotational) horizontal velocities and for vertical velocity $w$. The horizontal energy spectra are sketched following simulated and observed results (Chen and Tribbia 1981; Nastrom and Gage 1985; Skamarock et al. 2014). Because of Eq. (7), the slope of the $w$ spectrum equals the slope of the divergent energy spectrum plus two at large scales, and both spectra approach equal slopes at small scales. Any slope change in the divergent horizontal and the vertical velocity spectra occurs at about the same

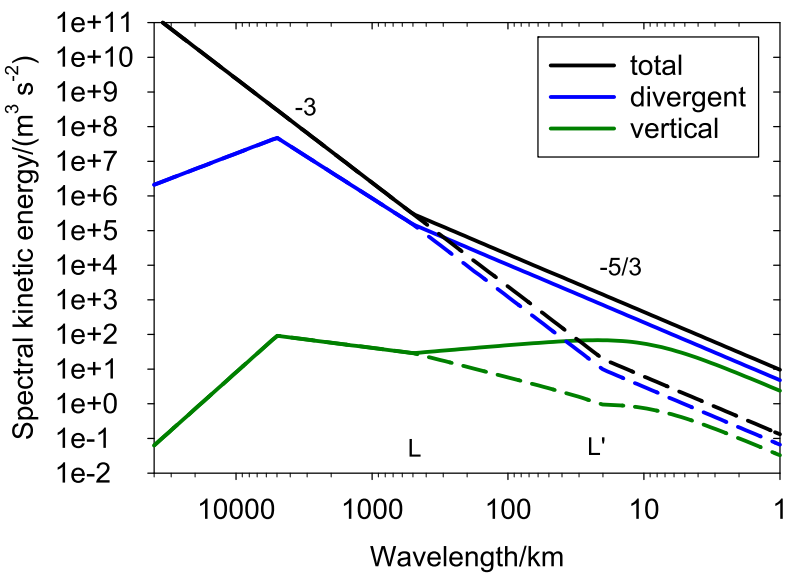

FIG. 1. Schematic of kinetic energy spectra of total and divergent horizontal velocities $E_{h}$ and $E_{d}$ and of vertical velocity $E_{w}$ for a case with transition from -3 to $-5 / 3$ spectra in $E_{h}$ at wavelengths $L=$ $500 \mathrm{~km}$ (solid lines) and at $L^{\prime}=20 \mathrm{~km}$ (dashed) with $h=10 \mathrm{~km}$, $\alpha=1 / 2$, and $\beta=0.11$.

transition scale $L$. The $w$ spectrum starts small at the longest wavelengths (near the Earth's circumference), may reach a local maximum at wavelengths $\lambda<L$ of baroclinic waves with local maximum in the spectrum of divergent velocities, gets flat for divergent energy spectrum with slope -2 , and approaches near isotropy at small scales. A maximum in $w$ spectra is to be expected at mesoscales if the divergent wind spectrum has a $-5 / 3$ slope, and such a maximum should be absent for a divergent wind spectrum steeper than -2 . For a wide $-5 / 3$ divergent spectrum, the vertical wind may reach a $1 / 3$ slope. The mesoscale maximum is higher and wider for larger spectral slope transition scale $L$ (solid curves) than for smaller $L^{\prime}$ (dashed curves). A more variable horizontal divergence spectrum may be accompanied by peaks in the $w$ spectrum. Broad maxima in the $w$ spectrum extending to the smallest mesoscales contribute strongly to the integral $\int E_{w} d k$ (i.e., total vertical velocity variance). In contrast, flat $w$ spectra at large scales (small $k$ ) contribute little to the total velocity variance.

It remains to be seen how well this hypothetical model fits reality in the vertical energy carrying scales. Since the horizontal spectra at one height $h$ cannot fully characterize the flow field below or above in its $3 \mathrm{D}$ details, we cannot expect that Eq. (7) is valid for individual cases. The given uncertainties become important in particular for low vertical correlations of the divergent horizontal motions at various heights, implying low $\beta$, and for events departing strongly from the assumed near isotropy at small scales. It will be interesting to see, how large the ensembles have to be to obtain statistically robust correlations. To test the hypothesis, we compare 
the "modeled spectrum" from Eq. (7) with reference results from previous numerical simulations in section 3 and with aircraft measurements in section 4 .

\section{Comparisons with global and regional simulated spectra}

The first test uses spectra derived with the Model for Prediction Across Scales (MPAS) in a global highresolution atmospheric simulation with $3 \mathrm{~km}$ grid scales (Skamarock et al. 2014). The published results include plots of 10-day-mean 2D energy spectra versus spherical wavenumber for the rotational, divergent and total kinetic energy from horizontal velocities and of the vertical velocity, separately in the troposphere $(h=$ $8.5-10.5 \mathrm{~km})$ and stratosphere $(16-18 \mathrm{~km})$, which we copied into Fig. 2. The top panel shows that the total kinetic energy spectra from MPAS exhibit the canonical spectrum structure with approximately -3 slopes at large scales and $-5 / 3$ slopes at mesoscales, making these spectra suitable for our study. The large-scale motions are dominated by energy from rotational motions, as expected (Gill 1982). The divergent part gets increasingly important at larger wavenumbers.

The divergent MPAS spectra are used as input for estimating the $w$ spectra, using Eq. (7) with $\alpha=1 / 2$ and $\beta=0.5$ and $0.05, h=9.5$ and $17 \mathrm{~km}\left(h_{\mathrm{eff}} \approx 5\right.$ and $\left.1 \mathrm{~km}\right)$, for the troposphere and stratosphere. For the selected $\beta$ values, the model and the MPAS spectra have about same integral energy $\int E_{w} d k$. The results are plotted together with the MPAS $w$ spectra in the bottom panel of Fig. 2. The modeled $w$ spectra show shapes similar to the reference spectra. In particular, both have a wide maximum at high wavenumbers where the divergent horizontal velocity spectrum has a rather flat slope, with steepness between -2 and $-5 / 3$. The modeled spectra also show, at least in the troposphere, a weak maximum value between global and synoptic scales near the spherical wavenumber 10 (wavelength $=2000 \mathrm{~km}$ ). The MPAS spectra decay rather steeply at high wavenumbers because of numerical dissipation. The modeled $w$ spectrum decays similarly. The model spectrum is weakly sensitive to $\alpha$ because of small $k h$ in the MPAS data. The stratospheric $\beta$ value is considerably less than one, as expected because the stratospheric wind is often weakly correlated with tropospheric winds. Perfect agreement could not be expected because the atmosphere is neither homogeneous nor horizontally isotropic. Also the model does not account for density variations and contributions from surface orography.

A second test uses spectra obtained from a regional numerical weather prediction model COSMO-DE applied for Europe (Selz et al. 2019) for a domain of about

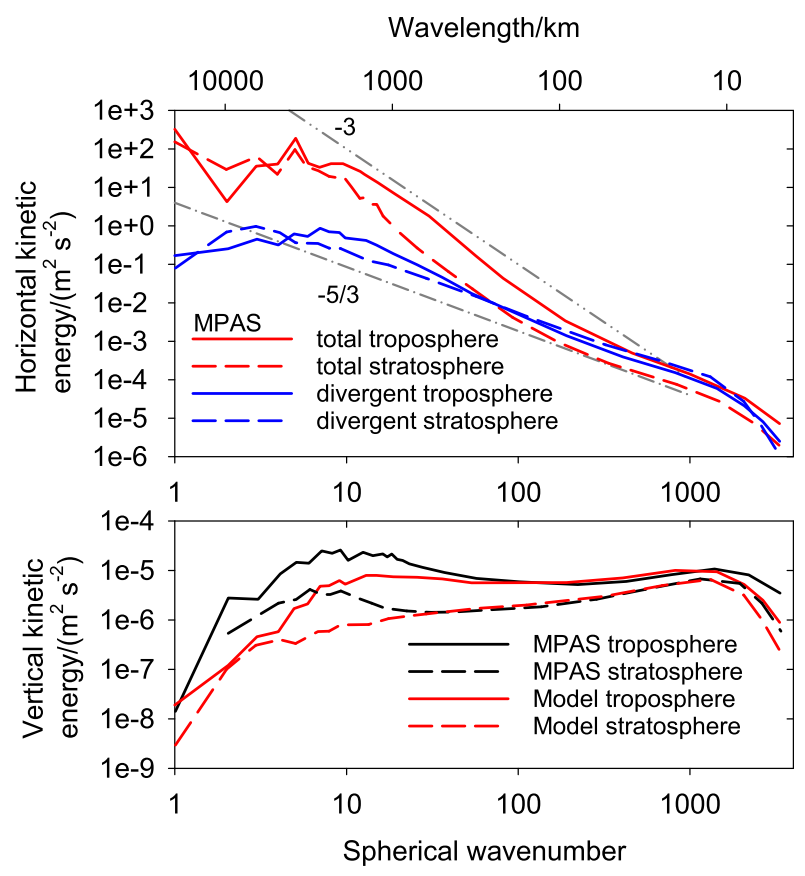

FIG. 2. (top) Energy spectra vs spherical wavenumber and wavelength for total (red) and divergent (blue) velocity components from the $3 \mathrm{~km}$ MPAS global simulation for the troposphere (solid) and the stratosphere (dashed), copied from Fig. 2 in Skamarock et al. (2014). Dashed-dotted lines represent the -3 and $-5 / 3$ slopes. (bottom) The $w$ spectra in the troposphere (solid) and stratosphere (dashed). The black curves are copies from Fig. 5 in Skamarock et al. (2014), and the red curves are from the present model, Eq. (7).

$1200 \mathrm{~km} \times 1300 \mathrm{~km}$ with $2.8 \mathrm{~km}$ horizontal resolution. The analysis data were obtained from the German Weather Service for 3 years, 2014-16, with 8 time steps per day at $3 \mathrm{~h}$ intervals, 8768 time steps in total. Mean 2D spectra of kinetic energy for 200, 300, and $500 \mathrm{hPa}$ have been extracted from the data by T. Selz (2019, personal communication), see Fig. 3. The total horizontal kinetic energy follows the canonical spectrum with about -3 slope at wavelengths $<200 \mathrm{~km}$ and slightly steeper than a $-5 / 3$ slope at wavelengths from about 20 to $100 \mathrm{~km}$. The kinetic energy is largest at $300 \mathrm{hPa}$. The spectrum of divergent motions follows a flatter spectrum, with slopes in between -2 and -1.8 between 2000 and $20 \mathrm{~km}$ wavelengths. The vertical kinetic energy spectrum is flat in that scale range, and decreases with slopes steeper than -3 between 20 and $10 \mathrm{~km}$. Spatial wavelengths less than about five grid spacings or $14 \mathrm{~km}$ may be not well resolved by the model (Craig and Selz 2018) and therefore are not shown. The fraction of divergent energy is small at large scales and reaches a maximum between 20 and $100 \mathrm{~km}$, exceeding $70 \%$ at $200 \mathrm{hPa}$ and $50 \%$ at lower heights. The model, Eq. (7), has been applied with the height corresponding to the pressure levels in the standard atmosphere. The parameter $\beta$ was selected 


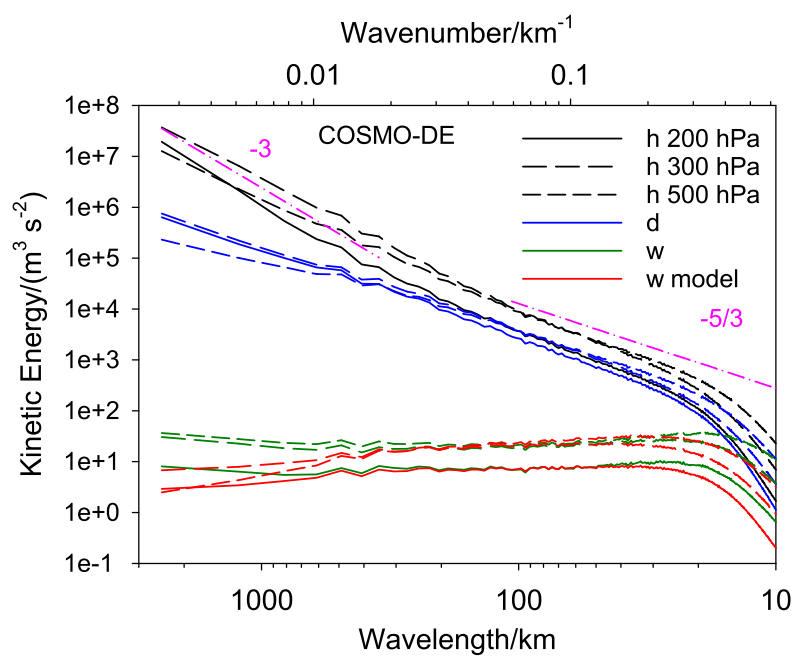

FIG. 3. Mean spectra of kinetic energy of total horizontal velocities $h$ (sum of divergent and rotational contribution), divergent horizontal velocities $d$, and vertical velocity $w$ at 200,300, and $500 \mathrm{hPa}$ (i.e., $11.8,9.2$, and $5.6 \mathrm{~km}$ height in the standard atmosphere) from 3 years of COSMO-DE data (Selz et al. 2019), courtesy of Tobias Selz. The dash-dotted lines are the -3 and $-5 / 3$ parts of the NGL spectrum The red curve is the model spectrum, Eq. (7), for $\alpha=1 / 2$ and $\beta=0.071,0.127$, and $0.229, h_{\text {eff }}=0.82,1.2$, and $1.3 \mathrm{~km}$, for 200,300 , and $500 \mathrm{hPa}$, respectively.

to best match the spectra at mesoscales. The data imply that the effective height $h_{\text {eff }}=\beta h$ decreases with height. The red curve in Fig. 3 shows that the model matches the COSMO-DE spectra over a wide range of scales (about $10-500 \mathrm{~km}$ ) not perfectly but within about $20 \%$ deviations. At large scales, the model expects a slight increase with wavenumber because the divergent spectra decrease less than quadratic, while the COSMO-DE $w$ spectra show a weak decrease, possibly because of the boundary conditions used in the regional model. Some of the model deviations may result from the orography, convection, and latent heat release, and the assumed 2D isotropy. The COSMO-DE $w$ spectra at mesoscales agree reasonably with the model and best at scales from about 20 to $500 \mathrm{~km}$. The basic hypothesis that $E_{w}$ is connected to $E_{d}$ at mesoscales by a factor $\left(h_{\mathrm{eff}} k\right)^{2}$ is supported by this comparison.

\section{Comparisons with aircraft measured mesoscale spectra}

\section{a. The aircraft measurements}

For comparison to observations, we use aircraft measurements from two field campaigns: The Deep Propagating Gravity Wave Experiment (DEEPWAVE; abbreviated D) provided $25 \mathrm{~Hz}$ wind and temperature data (https://doi.org/10.5065/D6BG2M1H) from 26 flights with the National Science Foundation Gulfstream GV research aircraft, operated by the National Center for Atmospheric Research, to observe gravity waves from mountains and tropospheric weather disturbances mainly in the lowermost stratosphere over New Zealand and the adjacent South Pacific (Fritts et al. 2016). The North Atlantic Waveguide and Downstream Impact Experiment (NAWDEX; abbreviated N) provided $10 \mathrm{~Hz}$ data (https://halo-db.pa.op.dlr.de/mission/93) from 13 flights with the German High-Altitude and Long-Range (HALO) Gulfstream G550 research aircraft, operated by the Deutsches Zentrum für Luft- und Raumfahrt, to observe weather systems in the upper troposphere and lower stratosphere in the North Atlantic, partly over Iceland and Greenland (Schäfler et al. 2018). The data from $\mathrm{D}$ and $\mathrm{N}$ contain, respectively, 98 and 44 straight and level flight sections longer than $2048 \mathrm{~s}$ (maxima of 6380 and $10553 \mathrm{~s}$, respectively); in total $120 \mathrm{~h}$, see the online supplemental material. Vertical velocity reached maximum values of 9.6 and $7.6 \mathrm{~m} \mathrm{~s}^{-1}$ for $\mathrm{D}$ and $\mathrm{N}$, with rms fluctuations of 0.45 and $0.25 \mathrm{~m} \mathrm{~s}^{-1}$, respectively. Fourier 1D power spectra are computed legwise by FFT after detrending with a linear least squares fit, and enforcing periodicity by a $10 \%$ Tukey filter (Sharman et al. 2014) from legs of equal duration selected from the flight sections, partially with $50 \%$ overlap. The number of equal-duration legs varies from 178 and 107 for 2048 s to 14 and 9 for 4096 s legs, for D and N, respectively. Taylor's hypothesis and the speed of the airplane $V$ relative to the air or ground are used to convert the measured time series into spatial records. In the standard analysis, $V$ is the leg-mean true airspeed TAS. Because of the large speed $\left(\sim 230 \mathrm{~m} \mathrm{~s}^{-1}\right)$, TAS and ground speed (GS) are not much different. The alongand transverse wind velocity components and their spectra $\left(E_{1}, E_{2}\right)$ are computed using leg-mean heading. Averaged angles (and heading instead of track angle) give less scatter than local values. As observed often (Schumann et al. 1995), low turbulence and strong stratification prevail in these data near the tropopause, with leg-mean Ozmidov scales $<10 \mathrm{~m}$, so that the inertial subrange of isotropic turbulence is not resolved with 10 or $25 \mathrm{~Hz}$ data (Lilly 1983; Riley and Lindborg 2008). Nevertheless, mean dissipation rates $\varepsilon$ are estimated to separate between high- and low-turbulence cases by fitting the Kolmogorov spectrum to the data. The fit uses data for frequencies from 0.27 to $1 \mathrm{~Hz}$ to avoid measurement noise at higher frequencies. Each set of legs is divided into two subsets with leg mean values below or above the median value of a selected parameter (e.g., for low and high $\varepsilon$ ). The spectra are spectral energy densities $S$ per wavenumber interval $d k$, in units of $\mathrm{m}^{3} \mathrm{~s}^{-2}$, with $\int S d k$ equal to the respective 
leg-mean energies. The potential energy spectrum $E_{p}$ is computed from half the temperature variance scaled with gravity, leg-mean temperature, and Brunt-Väisälä frequency; see Eq. (A2). Mean spectra are linear averages of the frequency spectra and converted to wavenumber spectra with $k=2 \pi f / V$ using the mean velocity $V$ on average over the leg subsets. Significant mean spectra, with $90 \%$ confidence limits (indicated by error bars in the plots) below about $20 \%$ at the largest scales, are obtained for $2048 \mathrm{~s}$ legs.

\section{b. Results and comparisons}

The observed spectra for both field campaigns (D and $\mathrm{N}$ ) and for low and high dissipation rates $\varepsilon$ are shown in Fig. 4 for a wide range in the mesoscales, with wavelengths $\lambda$ from $\sim 500$ to $0.1 \mathrm{~km}$. We selected the results for $2048 \mathrm{~s}$ legs because they are statistically robust and include data from most of the flights in the two campaigns (see supplemental material). Before we discuss the model performance for these observations, we inspect the spectra more generally.

The magnitudes and the shapes of the measured spectra differ between low and high $\varepsilon$ and $\mathrm{D}$ and $\mathrm{N}$. The $\mathrm{D}$ data have higher variances because of a larger fraction of flights in mountain waves. The spectral slope at $10-100 \mathrm{~km}$ wavelengths is -2.3 and -1.9 for low and high $\varepsilon$ for $\mathrm{D}$ and -2.2 and -2.0 for $\mathrm{N}$, steeper than $-5 / 3$. The mean spectra $E_{h}$ of horizontal velocities approach the Naström-Gage-Lindborg (NGL) 1D spectrum at low wavenumbers. The transition from -3 to flatter slopes occurs in the NGL spectrum near $\lambda \approx 460 \mathrm{~km}$ (see the appendix). So the slope of the NGL spectrum is already close to $-5 / 3$ in the scale range plotted. The Kolmogorov spectrum with the median dissipation rate derived from the data is about a factor 10 lower. We see that the separation into low and high $\varepsilon$ values causes a clear separation of the horizontal velocity spectra at small scales but little separation at large scales. Hence turbulence dissipation is not always the dominant parameter controlling mesoscale spectra; divergence of energy fluxes contribute also. The potential energy spectrum $E_{p}$ is parallel to but below the horizontal kinetic energy spectrum in the whole scale range. This will be discussed below. Both spectra seem to show two $-5 / 3$ ranges, one at lower and one at higher mesoscales, with an effective $\varepsilon$ about a factor of 10 higher at larger scales. For D, the large-scale part is even flatter than $-5 / 3$. Such a splitting was observed before (Dewan 1979; Jasperson et al. 1990; Callies et al. 2016), also by R. D. Sharman and R. G. Frehlich (2012, personal communication) who explained this splitting by energy transfer from nonlinear gravity waves. We see that the splitting is different for $\mathrm{D}$ and N. Possible reasons are discussed below.

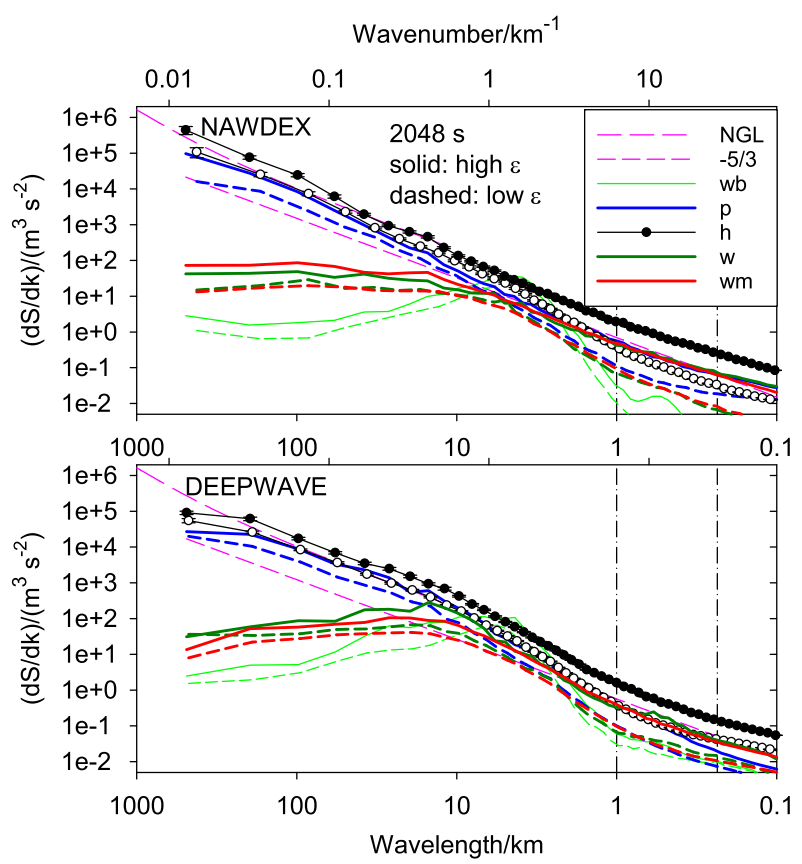

FIG. 4. Mean spectra of kinetic energy per wavenumber interval $d S / d k$ vs wavelength $\lambda$ and wavenumber $k=2 \pi / \lambda$, averaged over $20 \mathrm{log}$ intervals per decade at high wavenumbers, as derived from (top) 107 NAWDEX legs (N) and (bottom) 178 DEEPWAVE legs (D) of $2048 \mathrm{~s}$ duration each, for dissipation rates $\varepsilon$ below (dashed) and above (solid) the median values of 3.1 and $2.2 \times 10^{-6} \mathrm{~m}^{2} \mathrm{~s}^{-3}$ for $\mathrm{N}$ and $\mathrm{D}$, respectively, with $\varepsilon$ derived in the wavenumber interval indicated by vertical lines. Dashed purple lines represents the 1D Naström-Gage-Lindborg (NGL) spectrum (Lindborg 1999), and the $-5 / 3$ spectrum of Kolmogorov (1941) for median $\varepsilon$. Colored curves represent spectra of measured potential energy (blue), horizontal kinetic energy (from $u$ and $v$ ) with full and open symbols and error bars for confidence intervals (black), vertical air kinetic energy $w$ (dark green) and body kinetic energy $w_{b}$ (light green), and the modeled vertical air velocity spectrum $w_{m}$ (red). The model, Eq. (7), is applied for mean flight heights $h$ (11.7 and $12.2 \mathrm{~km}$ for the low and high $\varepsilon$ in D cases and 9.5 and $13.0 \mathrm{~km}$ for the low and high $\varepsilon$ in $\mathrm{N}$ cases), $d=1 / 2$, $\alpha=1 / 2$, and $\beta=0.11$.

The vertical aircraft body velocity spectrum shows a large peak, even exceeding horizontal velocity spectra, near $\lambda=5.0$ and $5.8 \mathrm{~km}$ for $\mathrm{N}$ and $\lambda=5.6$ and $6.7 \mathrm{~km}$ for $\mathrm{D}$, at low and high $\varepsilon$. This peak is a consequence of speed and autopilot-dependent mixed phugoid and pitch mode body oscillations (Nelson 1998), occurring in turbulent and in calm air. An analysis of these oscillations needs information about nonlinear contributions from the autopilot control loops that are not available publically. The vertical air velocity spectra are generally smooth over the whole scale range in spite of the large body motions, indicating precise measurements. Still, even the averaged air $w$ spectra show local maxima that may contain small contributions from uncertainties in the data. A more detailed analysis of these uncertainties is 
beyond the scope of this paper. From now on, $w$ spectra refer to vertical air motions.

We see that the $w$ spectra are flat at large scales, and decay about monotonously at small scales for $\mathrm{N}$, while the $w$ spectra for $\mathrm{D}$ data show a wide maximum at 19 and $15 \mathrm{~km}$ for high $\varepsilon$. Splitting the ensemble of legs into subsets with high and low $w$ variance, we find more pronounced maxima near about 10-20 km for higher $w$ variance. For lower $w$ variance, the spectra tend to get flatter without such local maximum. The $w$ spectra remain significantly (factor of 2-8) below the spectra of horizontal energy even at the smallest scales, without tendency to local isotropy.

It is remarkable that the model spectrum $E_{\mathrm{wm}}$ shows very similar behavior. The model spectrum $E_{\mathrm{wm}}$ is the red curve in Fig. 4. It got computed from the measured horizontal velocity spectrum and leg-subset mean heights $h$, Eq. (7), assuming $50 \%$ contributions from divergent velocities. The mean and standard deviations of the spectral ratio $E_{\mathrm{wm}} / E_{w}$ on average over all the bins shown are $1.12 \pm 0.55$ and $0.91 \pm 0.23$ for $\mathrm{D}$ and $1.06 \pm 0.16$ and $1.05 \pm 0.34$ for $\mathrm{N}$ for low and high $\varepsilon$, respectively. For a split of the legs into low and high flight levels relative to the local tropopause (from numerical weather prediction data), the ratio $E_{\mathrm{wm}} / E_{w}$ is shown in Fig. 5. The mean ratio values are $0.96 \pm 0.26$ and $0.96 \pm 0.31$ for $\mathrm{D}$ and $1.0 \pm 0.28$ and $1.05 \pm 0.24$ for $\mathrm{N}$ for TRO and STR in this figure. The ratio is remarkably close to one for both experiments with standard deviations below about $30 \%$ over the whole spectral range on average. The agreement would degrade, in particular at small scales, if we would replace $\alpha=1 / 2$ by larger values. Hence, the observations support $\alpha d=1 / 4$ more than $\alpha d=1$ or $4 / 7$ for linear inertial gravity waves or locally isotropic turbulence, respectively. As expected, $\beta$ and the effective height $h_{\text {eff }}$ may differ depending on the large-scale flow situation. For individual legs, the model/measurement ratio ranges between 0.2 and 5. The average ratio $E_{w m} / E_{w}$ is $1 \pm 0.3$. This is supported by analysis of spectra with other averaging times and other parameters used to separate leg subsets (besides dissipation $\varepsilon$ : vertical air and aircraft velocity variances, height above tropopause, wind speed, head wind, crosswind, a "waviness parameter" derived from cospectra and quadraure $w T$ spectra, Scorer parameter (Gisinger et al. 2017), spectral slope, and surface height).

The agreement is surprisingly good if one considers that these are $1 \mathrm{D}$ spectra, not $2 \mathrm{D}$, as assumed in the derivation of Eq. (7). The contributions from divergent and vertical waves perpendicular to the flight direction are not dominant at mesoscales in these measurements. The relatively small difference between $1 \mathrm{D}$ and $2 \mathrm{D}$

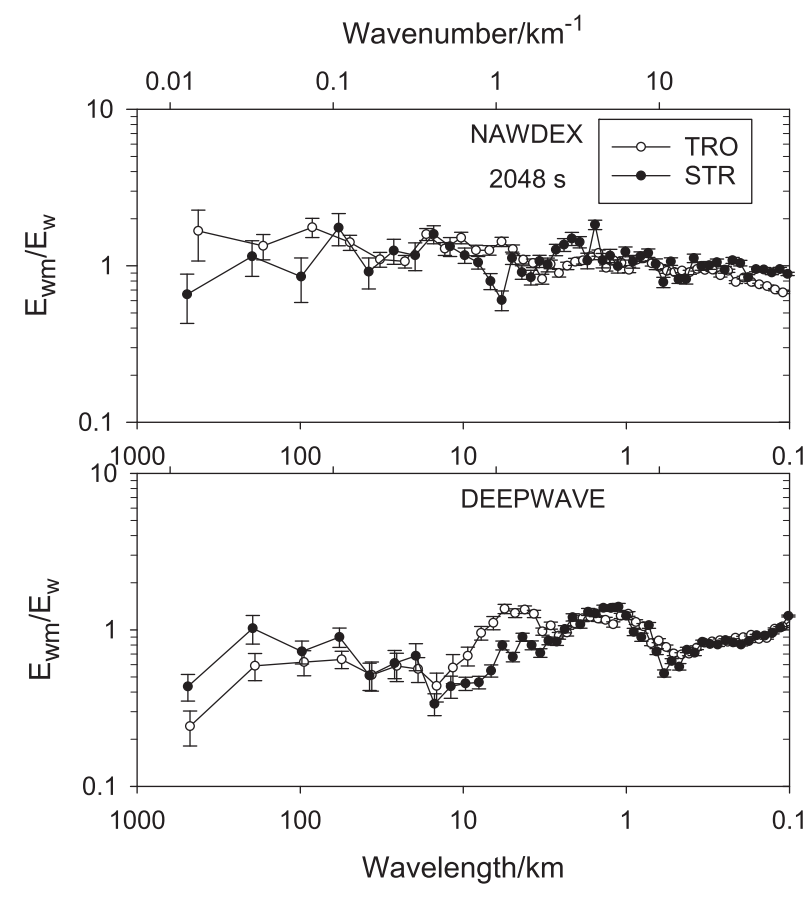

FIG. 5. Ratio of modeled to measured vertical kinetic energies for $2048 \mathrm{~s}$ legs from (top) $\mathrm{N}$ and (bottom) D for equal-sized subsets of legs with low and high height relative to the tropopause (TRO has open symbols; STR has full symbols).

spectra at mesoscales is also supported by model results (Zentek et al. 2016).

The degree of 2D isotropy may be estimated from Fig. 6 showing the fraction $E_{2} /\left(E_{1}+E_{2}\right)$ of spectral energies from transversal motions $E_{2}$ relative to longitudinal and transversal horizontal contributions. In 2D or 3D isotropic turbulence, 1D spectra of longitudinal (subscript 1) and transversal (subscript 2) velocities versus longitudinal wavenumber $k_{1}$ are related by isotropy and continuity [Lindborg's (2007) Eq. (38), and Hinze's (1959) Eqs. (3)-(71)] and for power-law spectra, $E(k) \sim k^{-n}, n \geq 1$, it follows that $E_{2} /\left(E_{1}+E_{2}\right)=3 / 4$ in 2D for $n=3$, and $E_{2} /\left(E_{1}+E_{2}\right)=4 / 7$ for $n=5 / 3$ in 3D. The colored lines in Fig. 6 show these values. We see that the data from $\mathrm{N}$ come reasonably close to isotropy for very large and very small wavenumbers, but the alongtrack variance dominates at mesoscales in these data. So the measured fields are not truly isotropic, which in fact could hardly be expected for a limited set of flights and for the likely nonisotropic nature of large-scale atmospheric dynamics. The data from $\mathrm{D}$ deviate from isotropy over the whole scale range. Low $E_{2} /\left(E_{1}+E_{2}\right)$ ratios may indicate enhanced $E_{1}$ from Doppler shifting in nonsteady irrotational gravity waves, which should increase with aircraft speed (Cho et al. 1999). However, the small-scale isotropy test gives about the same result regardless of whether the aircraft flew in or against the 


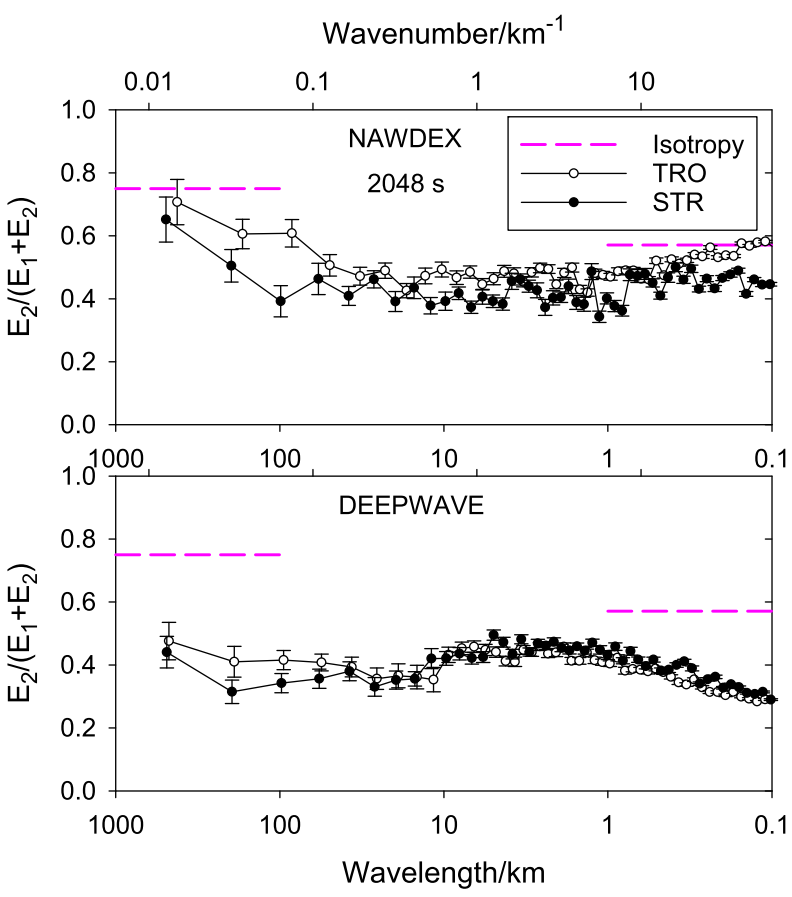

FIG. 6. Local isotropy test in terms of the ratio of horizontal transversal $\left(E_{2}\right)$ to sum of transversal and longitudinal $\left(E_{1}\right)$ velocity spectra for (top) N and (bottom) D and TRO (open) and STR (full), as in Fig. 5. The two horizontal purple lines represent the expected fractions in cases of locally isotropic and incompressible flow in 2D turbulence with a -3 slope at large scales and in 3D turbulence with a $-5 / 3$ spectrum at small scales.

main wind direction or perpendicular to it, so that measurement issues cannot be excluded.

The assumption that the divergent part amounts to $50 \%$ in the mesoscales needs some checking and is of general interest. In spite of the obvious deviations from isotropy and unknown deviations from 2D turbulence, the measured $E_{1}$ and $E_{2}$ spectra are used, as in previous studies (Bierdel et al. 2016; Callies et al. 2016; Li and Lindborg 2018), to estimate the divergent $\left(E_{d}\right)$ and rotational $\left(E_{r}\right)$ horizontal kinetic energy spectra with the Helmholtz decomposition for 2D isotropic turbulence of Lindborg (2015). Previous studies found divergence fractions from $1 / 3$ to $2 / 3$ ( $d / r$ ratios from $1 / 2$ to 2 ), varying among the datasets used but tending to higher values in the stratosphere where high-frequency wavy motions dominate (Blažica et al. 2013; Bierdel et al. 2016; Callies et al. 2016; Li and Lindborg 2018). The results in Fig. 7 reveal spectral divergence energy fractions varying with wavenumber and datasets. The $\mathrm{N}$ data show a more uniform behavior and suggest a near $40 \%$ fraction in the troposphere and near $70 \%$ in the stratosphere. For D, these results are more variable; here, the method partially fails to provide physically reasonable results. The derived $E_{r}$ values are partly negative because of

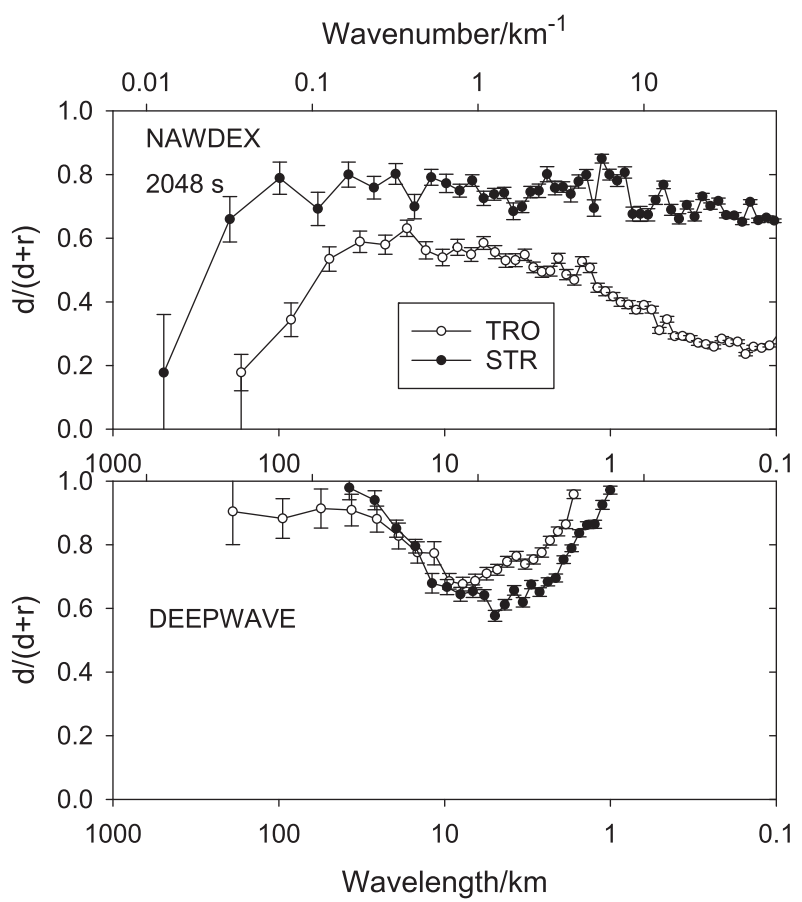

FIG. 7. Fraction of divergent horizontal kinetic energy, for (top) $\mathrm{N}$ and (bottom) D, and TRO (open) and STR (full), as in Fig. 5.

negative $E_{2}-E_{1}$ contributions (not plotted). Overall, the data roughly support the assumption of $d$ near $50 \%$ at mesoscales.

The ratios $E_{h} / E_{p}$ and $E_{w} / E_{p}$ of horizontal and vertical kinetic energies relative to potential energy are shown in Fig. 8 and compared with the 1D spectral model derived in the appendix. Equations (A4) and (A5) describe the ratio of potential energy to horizontal and vertical motions from linear gravity waves for given empirical spectra $E_{h}$ and $E_{w}$. As input, we use the 2D spectrum, Eq. (A6), as in Lindborg (1999), with transition scale $L=394 \mathrm{~km}$, estimate the fraction of divergent energy from Eq. (A8), and estimate $E_{w}$ from Eq. (7) with $h$ from the data, $\beta=0.11$, and $\alpha=1 / 2$. The blue and red lines show the 1D spectra computed numerically from the $2 \mathrm{D}$ spectra. The data show a ratio $E_{h} / E_{p}$ between 2 and 8 . A ratio of $E_{h} / E_{p}>1$ is expected for linear gravity waves and $E_{h} / E_{p} \approx 2$ for geostrophic turbulence (Charney 1971; Gage and Nastrom 1986). The model based on linear gravity waves underestimates $E_{h} / E_{p}$, by at least a factor of 2, presumably because of missing rotational wind components. The ratio $E_{w} / E_{p}$ starts from very small values at global scales, increases about quadratically for scales $>L$, and approaches one within about $20 \%$ deviations in the data for small scales $\lambda<L$. At small scales, values of $E_{w} / E_{p}>1$ occur, which are inconsistent with linear gravity waves and hence either nonlinear waves or turbulent motions. Generally, the 


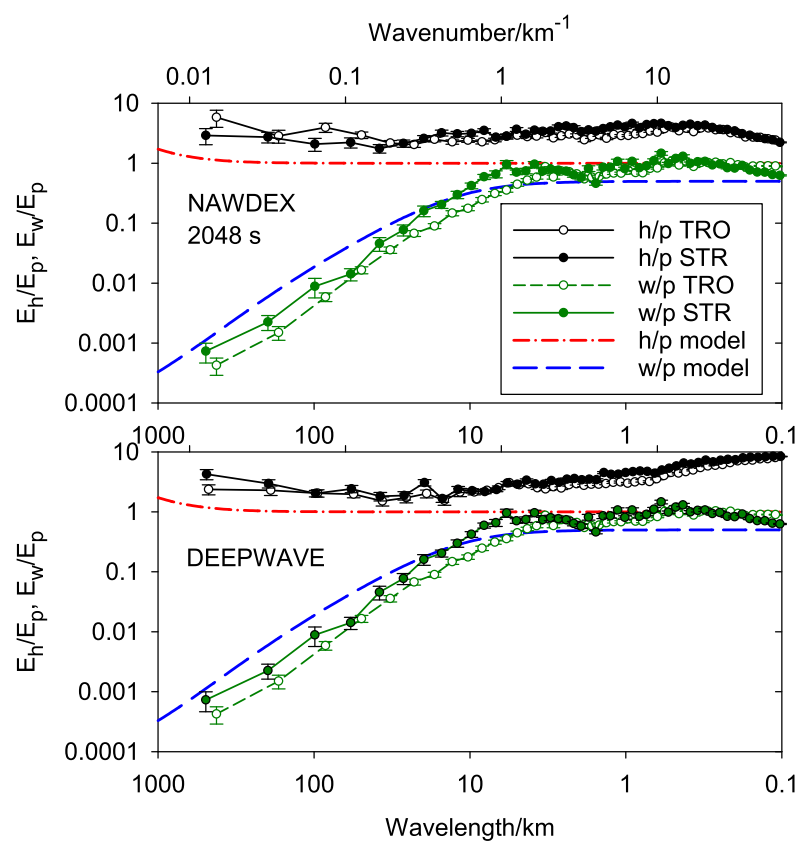

FIG. 8. Ratio of horizontal to potential energy $E_{h} / E_{p}$ and of vertical to potential energy $E_{w} / E_{p}$, for (top) $\mathrm{N}$ and (bottom) $\mathrm{D}$ and TRO (open) and STR (full), as in Fig. 5. The red and the blue dashdotted curves represent the gravity wave model for $E_{h} / E_{p}$ and $E_{w} / E_{p}$; see Eqs. (A5)-(A8) and (7).

data show smooth changes with $\lambda$ as modeled. This also supports the measurement quality.

In Fig. 9, results are compared for flights over mountains (land) and over oceans (zero surface height above sea level and more than $300 \mathrm{~km}$ distance from the geographic centers of New Zealand and Iceland). The results differ in that the splitting into two $-5 / 3$ spectra seems to be stronger over mountains than over oceans. The spectral energies are definitely higher over mountains than over oceans, as found earlier (Nastrom et al. 1987). The ratio $E_{\mathrm{wm}} / E_{w}$ and, hence, the relationship between the horizontal and vertical spectra, differs only little between ocean and mountain cases.

Finally, Fig. 10 shows the spectra for the longest legs. Here, the wavelengths reach 1500 and $2000 \mathrm{~km}$ for D and $\mathrm{N}$. We do not know of similarly long legs with $w$ spectra from the literature. At the largest scales, the spectrum is based on just one wave, but the results get significant for $\lambda<300 \mathrm{~km}$. The $\mathrm{N}$ data exhibit a significant $-5 / 3$ slope over three decades of scales, which is remarkable. The $\mathrm{D}$ spectra show again indications for two $-5 / 3$ ranges. The results suggest a transition scale $L$ of order $300 \mathrm{~km}$ for $\mathrm{N}$ with rather low $w$ variance. For the more turbulent $\mathrm{D}$ case, this transition seems to occur at larger wavelengths, possibly above $1500 \mathrm{~km}$. Hence, the slope transition in the canonical spectrum varies depending on vertical velocity

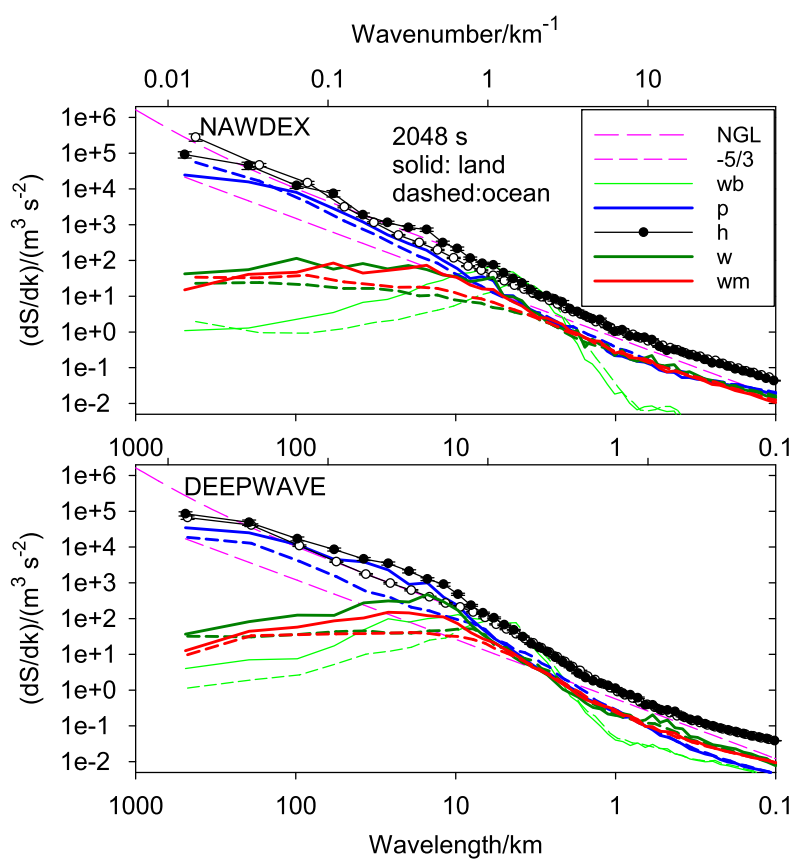

FIG. 9. As in Fig. 4, but separately for ocean (dashed) and land (solid). The averages include 125 (87) ocean and 53 (20) land legs for D (N) of $2048 \mathrm{~s}$ duration each. The mean flight heights are $h=$ 11.7 and $12.2 \mathrm{~km}$ for D over ocean and land and $h=9.6$ and $13.0 \mathrm{~km}$ for $\mathrm{N}$ over ocean and land. The mean ratio $E_{\mathrm{wm}} / E_{w}$ is $0.94 \pm 0.26$ and $0.91 \pm 0.31$ for $\mathrm{D}$ and $1.06 \pm 0.28$ and $0.87 \pm 0.28$ for $\mathrm{N}$.

variance at mesoscales. The modeled $w$ spectrum (red curve) is close to the measured $w$ spectrum (green) at least for scales smaller than about $200 \mathrm{~km}$ in both datasets.

\section{Discussion and implications}

The comparisons to global and regional models and to measurements show that the model is useful in characterizing the horizontal and vertical atmospheric flow energetics. The comparison with $2 \mathrm{D}$ spectra from a global model and a regional model confirm Eq. (7) in approximating the connection between $E_{d}$ and $E_{w}$ reasonably, at least in the mesoscales. The value of $h_{\mathrm{eff}} / h$ is smaller for the stratosphere than the troposphere because of small vertical correlations of horizontal motions in the stratosphere with the flow farther down, in particular, below the tropopause. The comparisons with observed 1D spectra at mesoscales near the tropopause suggest that Eq. (7) describes the relationship between measured spectra on average to an accuracy of about $30 \%$. The relationship is best applicable for spectra on average over many long flights. Apparently the number of flights in N and D, 107 and 178 for 2048 s leg lengths, were just sufficient in this sense. But also individual legs, like the longest flights of each campaign, with 


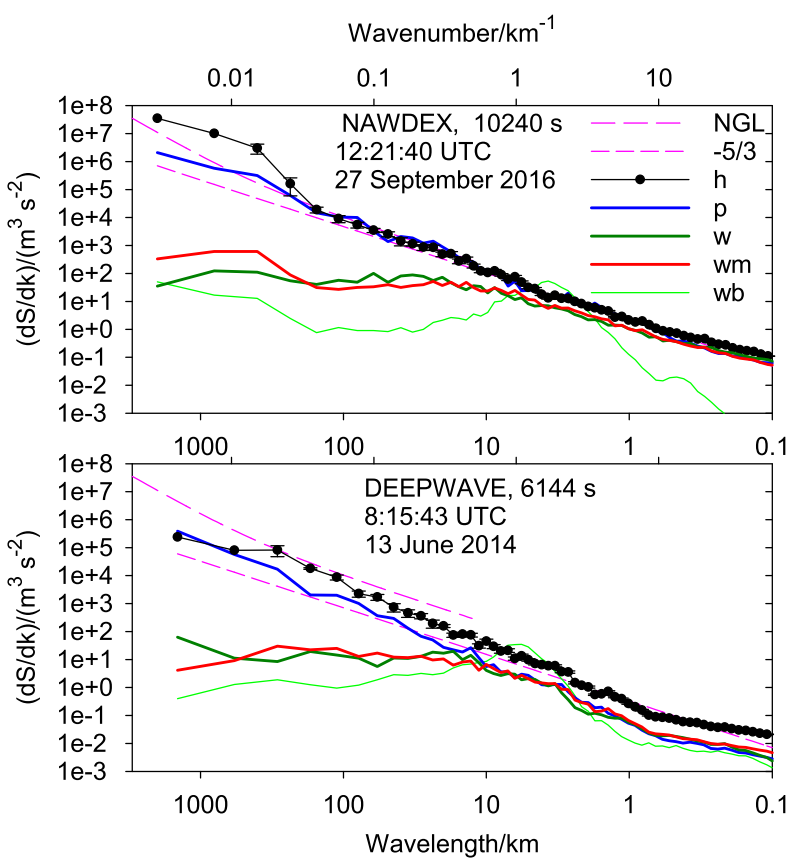

FIG. 10. As in Fig. 4, but for the longest legs of (top) $10240 \mathrm{~s}$ $\left(2000 \mathrm{~km}\right.$ at $h=8.7 \mathrm{~km},\left\langle w^{\prime 2}\right\rangle=0.024 \mathrm{~m}^{2} \mathrm{~s}^{-2}, \varepsilon=17 \times 10^{-6} \mathrm{~m}^{2} \mathrm{~s}^{-3}$ $N=0.012 \mathrm{~s}^{-1}$, and $4.2 \mathrm{~km}$ below the tropopause) for $\mathrm{N}$ and (bottom) $6144 \mathrm{~s}\left(1500 \mathrm{~km}\right.$ at $h=12.3 \mathrm{~km},\left\langle w^{\prime 2}\right\rangle=0.068 \mathrm{~m}^{2} \mathrm{~s}^{-2}, \varepsilon=1 \times$ $10^{-6} \mathrm{~m}^{2} \mathrm{~s}^{-3}, N=0.0266 \mathrm{~s}^{-1}$, and $1.2 \mathrm{~km}$ above the tropopause) for D. The leg start times are given. The values of $E_{\mathrm{wm}} / E_{w}$ are $1.41 \pm 0.7$ for $\mathrm{D}$ and $1.18 \pm 1.2$ for $\mathrm{N}$.

$1500-2000 \mathrm{~km}$ leg lengths, support the model within reasonable deviations.

Based on the $10 \%$ and $90 \%$ limits of the $w$ energy distribution shown in Fig. 11, most of the vertical velocity energy near the tropopause occurs at mesoscales between about 0.5 and $80 \mathrm{~km}$, with $50 \%$ at scales between 7 and $17 \mathrm{~km}$. The result confirms that uncertainties in Eq. (7) originating from processes at large scales have little impact on the integral $w$ variances. The global MPAS model with a $3 \mathrm{~km}$ grid resolves $50 \%$ contributions at scales below about $25 \mathrm{~km}$. Apparently, grid resolution has still to be increased by factors of $3-10$ to resolve $50 \%-90 \%$ of the $w$ variances near the tropopause. An analysis of large-eddy simulations, as, for example, in Heinze et al. (2017), resolving mesoscale and turbulence scales, may be used to check these results.

The model derivation ignored density variations and variable surface orography, which are of course important for the atmosphere. For variable surface height, as in the mountain waves observed during DEEPWAVE, mountains likely add a contribution depending on the surface slope, horizontal velocity near the surface, and vertical wave propagation. Orography certainly induces

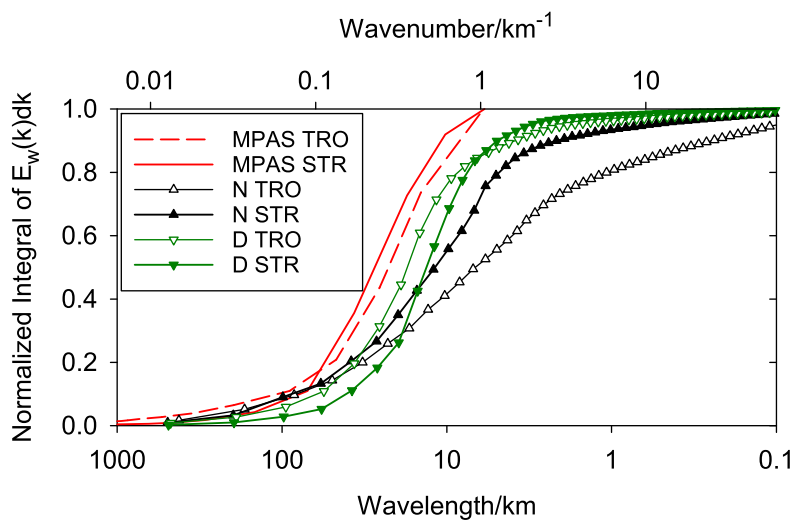

FIG. 11. Normalized integral of vertical kinetic energy, $\int_{k_{\min }}^{k} E_{w}\left(k^{\prime}\right) d k^{\prime} / \int_{k_{\min }}^{k_{\max }} E_{w}\left(k^{\prime}\right) d k^{\prime}$, vs wavenumber $k$ from numerical results (MPAS; Skamarock et al. 2014) and from measurements (N and D) on $2048 \mathrm{~s} \mathrm{legs,} \mathrm{for} \mathrm{tropospheric} \mathrm{(TRO)} \mathrm{and} \mathrm{stratospheric}$ (STR) cases. $50 \%$ of the energy is included for wavelengths $\lambda=2 \pi / k$ smaller than 23,7 , and $17 \mathrm{~km}(29,12$, and $14 \mathrm{~km})$ for MPAS, N, and D in TRO (STR), respectively. The MPAS data include resolved $w$ energy globally above $6 \mathrm{~km}$ scales. The data for $\mathrm{D}$ and $\mathrm{N}$ resolve scales from $\sim 480 \mathrm{~km}$ down to 18 and $46 \mathrm{~m}$, respectively, in this analysis.

large motion changes regionally, but mean $w$ spectra may be less affected after averaging over a few long waves at small $k$ and over the many short waves that occur at high $k$. Density variations from pressure changes and internal heat sources contribute to vertical velocity changes that we cannot quantify (Malardel and Wedi 2016; Craig and Selz 2018; Egger and Hoinka 2019). In the anelastic approximation, $w$ would be the vertical wind weighted with density at height $h$, and $\bar{u}$ and $\bar{v}$ the vertically averaged horizontal mass fluxes. Here, surface contributions may get overestimated because of strong decrease of density with height (Egger and Hoinka 2019). Analyzing the flight data weighted with the measured densities shows small changes in the $E_{\mathrm{wm}} / E_{w}$ ratio, but the data contain only a small spread of heights. Separation of the spectra into those over land and ocean has far larger impact.

The kinetic relationship implies a connection between the spectral slope of the horizontal velocities and the vertical velocity spectrum at least for incompressible and horizontally isotropic divergent motions. Besides in the numerical results shown in section 3, this relationship can also be identified in $\mathrm{w}$ and divergence spectra derived from numerical simulations of baroclinically unstable shear flows (Menchaca and Durran 2019). This kinematic connection may cause a dynamic connection between vertical and horizontal motions and affect the transition scale $L$. The flatter spectrum at mesoscales may be a consequence of energy transfer from horizontal to vertical divergent motions. This would be 
consistent with studies noting that the transition in spectral slope of kinetic energy spectra occurs when the divergent component of the spectrum becomes similar in magnitude to the rotational component (Waite and Snyder 2009; Skamarock et al. 2019). Our data suggest that the spectral energy transfer rate is not only controlled by the classical $\varepsilon$. Other energy sources and losses at mesoscales may be more important than turbulence at the same level (e.g., by blocking and vortex shedding because of convection and mountains and by latent heat release in clouds) (Lilly 1983; Craig and Selz 2018). Note that spectral energy exchange between large and small scales occurs at time scales of order $E / \varepsilon$, which may be large compared to advection time scales $L / U$ (e.g., over mountains with width $L$ and wind speed $U$ ). Hence, energy maxima in the spectra at certain scales may originate mainly from energy induced by ambient disturbances (mountains, convection, etc.) at the same scales and less by upscale or downscale energy transfer.

We note that similar spectral relations with $E_{w} \approx(h k)^{2} E_{h}$ for 2D spectra, have been found also for the boundary layer close to the Earth's surface (Peltier et al. 1996; Kang and Lenschow 2014; Tong and Nguyen 2015). The authors explained the connection with continuity as we do, with different quantitative models. Also other observations show similar spectral relationships (Gultepe and Starr 1995; Bacmeister et al. 1996; Gao and Meriwether 1998). A quadratic relationship between $w$ and $u$ spectra at low wavenumbers is also detectable in $w$ spectra derived from potential temperature changes (Nastrom and Fritts 1992). Even strong downslope wind spectra show such behavior (Lilly 1978). The $w$ spectra peaks at $4-16 \mathrm{~km}$ wavelengths obtained and analyzed by Zhang et al. (2015) and Callies et al. (2016) cannot be explained this way because the peaks have no counterparts in the measured horizontal velocity and potential energy spectra. There must have been other sources causing such vertical wind signals, such as divergent motions perpendicular to the flight direction (e.g., when passing mountain valleys with variable cross-stream winds) (Smith et al. 2016). Our results show that the smooth maximum of vertical velocity kinetic energy found in aircraft measurements at $5-15 \mathrm{~km}$ horizontal scales has good reasons. This supports the validity of such measurements. This is important to know when using long-range and high-speed research aircraft for atmospheric research.

\section{Conclusions}

A hypothesis has been introduced that connects vertical and horizontal divergent motions by mass continuity at large scales and by a trend toward locally isotropic gravity wave or turbulence dynamics at small scales. The hypothesis is expressed in terms of a simple algebraic relationship between the horizontal spectrum of vertical velocities $E_{w}$ at a given height $h$ and the spectra of total and divergent horizontal winds at the same level, $E_{h}$ and $E_{d}$. For large scales, $E_{w}$ is close to $\left(h_{\mathrm{eff}} k\right)^{2} E_{d}$ where the effective height is related to geometric height by $h_{\mathrm{eff}}=\beta h$, and $\beta$ is a parameter of order 0.1 depending on vertical correlations between horizontal divergent motions. Comparisons with simulated and measured spectra, for a wide range of atmospheric conditions, in different parts of the world and for different measurements, support the hypothesis at mesoscales within about $30 \%$. The small divergent motions at large scales and the high anisotropy at small scales differ from expectations for linear gravity waves. The ratio of vertical kinetic energy to potential energy increases quadratic with wavenumber at large scales and approaches a constant ratio at small scales but the ratio is larger than expected for linear gravity waves. Hence, the spectra cannot be explained by inertia-gravity waves, consistent with earlier findings.

The relation used relies on 2D isotropy, near incompressible continuity, and small surface contributions. These restrictions may be overcome with more extended theories and more knowledge on vertical correlations and anisotropy of horizontal divergent velocity components.

Spectra of vertical velocity and of potential energy are important additions to spectra of horizontal kinetic energy to characterize the atmospheric flow energetics. The near kinematic connection between vertical and horizontal divergent motions and with buoyancy likely has dynamical implications by causing energy transfer between horizontal and vertical motions. Further studies may quantify this energy transfer. Energy exchange between horizontal and vertical motions may be the key for understanding the split into divergent and rotational motions and the related flattening or steepening of the spectrum in stably stratified turbulence.

We used $120 \mathrm{~h}$ of constant-level flight data in the upper troposphere and lower stratosphere at scales from 0.1 to $2000 \mathrm{~km}$ horizontally from the DEEPWAVE and NAWDEX flight measurements. DEEPWAVE concentrated on mountain waves near New Zealand while NAWDEX addressed warm conveyer belts and frontal processes in the North Atlantic. In spite of these differences, the wind and temperature spectra from these experiments show remarkable similarities. The resolved smallest motions measured in mostly stably stratified layers near the tropopause show ratios $E_{w} / E_{h}$ of about $0.1-0.5$, far less than for isotropic turbulence. The horizontal divergence fraction was found to be $40 \%-70 \%$ 
with higher values in the stratosphere, similar to earlier results. The data show significant deviations from $2 \mathrm{D}$ isotropy. Horizontal energy spectra with $-5 / 3$ slopes are observed in the troposphere over oceans. Near the tropopause, spectra over mountains are often steeper than over oceans, possibly with two $-5 / 3$ subranges. The mesoscale $-5 / 3$ spectrum is not controlled by local energy dissipation alone. Instead, energy exchange with equal-scale disturbances (e.g., by vertical motions) may be more important. The increase of vertical velocity kinetic energy over a broad range of scales over mountains is consistent with a flattening of the spectrum of horizontal velocity kinetic energy in the measurements.

Acknowledgments. Part of this research was funded within the German research initiative "Role of the Middle Atmosphere in Climate" (ROMIC/01LG1206A) by the German Ministry of Research and Education in the project "Investigation of the Life Cycle of Gravity Waves" (GWLCYCLE). The author gratefully acknowledges steady support by Markus Rapp, stimulating and long-term discussions with Andreas Dörnbrack and Robert Sharman, specific contributions from Martina Bramberger, Sonja Gisinger, Chris Kruse, Andreas Schäfler, Nils Wedi, Erik Lindborg, George Craig, and Tobias Selz, and valuable comments by Thomas Birner and Joseph Egger. The flight data were provided within the DEEPWAVE and NAWDEX projects by the National Center for Atmospheric Research and the Flight Facility of the Deutsches Zentrum für Luft- und Raumfahrt.

\section{APPENDIX}

\section{Model Equations for Linear Inertia-Gravity Waves and Modifications}

This appendix relates various forms of kinetic and potential energy and tests their consistency with linear hydrostatic inertia-gravity wave theory. Linear inertiagravity waves can be expressed as Fourier modes as a function of horizontal and vertical wavenumbers $k_{x}$ and $k_{y}, m$, and the intrinsic frequency $\hat{\omega}$ (Gill 1982; Fritts and Alexander 2003). For $f<\hat{\omega}<N$, these scales satisfy the well-known dispersion law:

$$
\hat{\omega}^{2}=f^{2}+\frac{N^{2}\left(k_{x}^{2}+k_{y}^{2}\right)}{m^{2}},
$$

with Brunt-Väisälä frequency $N$ and Coriolis parameter $f$. On average over wave phases, such waves have vertical energy $W$, horizontal kinetic energy $K$, and potential energy $P$,

$$
W=\frac{1}{2} \overline{w^{2}}, \quad K=\frac{1}{2} \overline{u^{2}+v^{2}}, \quad P=\frac{1}{2} \frac{g^{2}}{N^{2} T_{0}^{2}} \overline{T^{\prime 2}},
$$

with gravity $g$ and mean temperature $T_{0}$, with energy ratios (Geller and Gong 2010):

$$
\frac{K}{P}=\frac{1+(f / \hat{\omega})^{2}}{1-(f / \hat{\omega})^{2}} \text { and } \quad \frac{W}{P}=\frac{\hat{\omega}^{2}}{N^{2}} .
$$

Observations of measured energy ratios and frequency spectra are consistent with these relationships (Podglajen et al. 2016). As a consequence of Eq. (A3), hydrostatic linear gravity waves require $W<P<K$ because otherwise the intrinsic frequency is outside the range $f<\hat{\omega}<N$. The equations also imply $W / K \rightarrow 0$ for $\hat{\omega} \rightarrow f$ and $W / K \approx 1$ for $\hat{\omega}=N \gg f$. For nonhydrostatic gravity waves, these ratios depend additionally on vertical wavenumber $m$ (Geller and Gong 2010).

From the hydrostatic equations one can eliminate the intrinsic frequency $\hat{\omega}$ to derive

$$
\frac{W}{P}=\frac{f^{2}}{N^{2}} \frac{K+P}{K-P}
$$

$$
\frac{P}{K}=-\frac{1+\frac{W}{K}\left(\frac{N}{f}\right)^{2}}{2}+\left\{\left[\frac{1+\frac{W}{K}\left(\frac{N}{f}\right)^{2}}{2}\right]^{2}+\frac{W}{K}\left(\frac{N}{f}\right)^{2}\right\}^{1 / 2}
$$

Equation (A4) allows computing $W$ for given $K$ and $P$. The result also satisfies Eq. (A5). Since the model is linear, the relationships hold for each Fourier mode $k$ in terms of spectral energy densities $E(k): P=E_{p} d k$, $W=E_{w} d k$, and $K=E_{h} d k$.

Let us assume, Eq. (7) may be used to compute $E_{w}$ for given $E_{d}$. For completion, we then need $E_{h}$ and $E_{d}$. We may assume that the spectrum $E_{h}$ of total horizontal kinetic energy follows approximated measured spectra (Lindborg 1999, 2009),

$$
E_{h}=a k^{-3}+b k^{-5 / 3} .
$$

For 1D and 2D spectra, the coefficients are $a_{2 \mathrm{~d}}=2 a_{1 \mathrm{~d}}$, $b_{2 \mathrm{~d}}=1.4 b_{1 \mathrm{~d}}, a_{1 \mathrm{~d}}=9.1 \times 10^{-4}$, and $b_{1 \mathrm{~d}}=3 \times 10^{-10}$ (coefficients in units of meters and seconds). In log scales, the asymptotic -3 and $-5 / 3$ lines cross at the transition scale $L$ :

$$
L=\frac{2 \pi}{\exp [(3 / 4)(\ln b-\ln a)]} .
$$

The value of $L$ is $457 \mathrm{~km}$ for the $1 \mathrm{D}$ and $394 \mathrm{~km}$ for the $2 \mathrm{D}$ spectra. Finally, we need $E_{d}$. In view of observations, 
it is tempting to assume that the fraction of divergent motions is close to $d$ of order $1 / 2$ at scales $<L$, and approaches zero at scales $>L$, and varies with $k$ like

$$
\frac{E_{d}}{E_{h}}=d \frac{k L}{1+k L} .
$$

A value of zero for $E_{d} / E_{h}$ at large scales is consistent with quasigeostrophic theory [see section 8.16 in Gill (1982)] and a linear relationship between $E_{d}$ and $E_{h}$ at $k \ll L$ would be consistent with the -3 slope of $E_{h}$ and the -2 slope of $E_{d}$ in observations (Chen and Tribbia 1981) and numerical results (Skamarock et al. 2014). However, for linear gravity waves, $E_{d} / E_{h}$ is not a free parameter; it follows from the equations of motions.

Along the same line of arguments as in Gill (1982, section 8.4 therein), for the hydrostatic case, one can derive the mean horizontal kinetic energy of divergent motions $D=(1 / 2)\left(\overline{u_{d}^{2}+v_{d}^{2}}\right)$. For this purpose, we start from the linearized Boussinesq equations, apply the Helmholtz decomposition to split the velocities into rotational $\left(u_{r}, v_{r}\right)$ and divergent velocities $\left(u_{d}, v_{d}\right)$, and determine the related streamfunction and velocity potential such that their Laplacians equal the $2 \mathrm{D}$ divergence and vorticity. This allows computing $\left(u_{d}, v_{d}\right)$ and the mean ratio of energies of divergent motions $D$ relative to vertical energy $W$ and to total horizontal energy $K$ :

$$
\frac{D}{W}=\frac{m^{2}}{k^{2}+l^{2}} \text { and } \quad \frac{D}{K}=\frac{1}{1+(f / \hat{\omega})^{2}} .
$$

The rotational energy equals $K-D$. The first part would be about consistent with Eq. (7) for $\beta \approx 1 / \pi$, if applied to vertical wavenumbers $m \approx \pi / h$. However, the second equation implies that $D / K$ varies between 0.5 at low frequencies and 1 at high frequencies $\hat{\omega} / f$. In fact, replacing Eq. (A8) by $E_{d} / E_{h}=0.5\left\{1+(2 k L)^{2} /\left[1+(2 k L)^{2}\right]\right\}$ gives results consistent with linear gravity waves but inconsistent with observations and global numerical results.

Hence, as found earlier (Lindborg 2007), the theory of linear gravity waves does not explain the observations. Still, Eqs. (7) and (A4)-(A8) provide a closed set of model equations, which are interesting to consider. In this model, only $E_{p}$ is related to linear gravity waves, Eq. (A5), and the low-wavenumber part should be taken with care. The value of $E_{p}$ is sensitive to the values of $f$ and $N$; that is, $E_{p}$ increases with increasing $N / f$. Typical values at midlatitudes near the tropopause are $f=10^{-4} \mathrm{~s}^{-1}$ and $N=0.02 \mathrm{~s}^{-1}$. Moreover, the results depend on $\alpha$ and $\beta$. The wave theory suggests $\alpha=1 / d$ because then $E_{w} \approx$ $E_{p} \approx E_{h}$ for $\hat{\omega}=N \gg f$. However, the observations in stratified turbulence, for example, those shown in section 4 , show significantly smaller ratios of $E_{w} / E_{h}$.

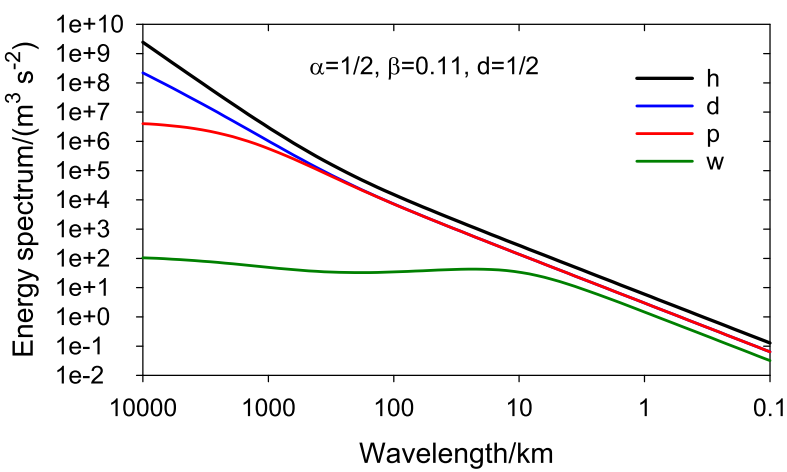

FIG. A1. The 2D spectra of total, divergent, vertical, and potential energy ( $E_{h}, E_{d}, E_{w}, E_{p}$, respectively) vs wavenumber $k$ and wavelength $\lambda=2 \pi / k$, for prescribed $E_{h}$, modeled $E_{d}$ and $E_{w}$, and $E_{p}$ derived for linear hydrostatic inertia-gravity waves, for fixed values of $\alpha, \beta$, and $d$ (see figure for values) and for $h=10 \mathrm{~km}$.

Therefore, we leave the wave theory and assume $d=1 / 2$, $\alpha=1 / 2$, and $\beta \approx 0.11, h=10 \mathrm{~km}$. For these parameters, the spectra are shown in Fig. A1. For comparison with the aircraft measurements, we need the $1 \mathrm{D}$ spectra. The $1 \mathrm{D}$ spectra can be obtained from the given 2D spectra:

$$
E_{1 \mathrm{~d}}\left(k_{1}\right)=\frac{2}{\pi} \int_{0}^{\infty} \frac{E_{2 \mathrm{~d}}(k)}{k} d k_{2}, \quad k=\left(k_{1}^{2}+k_{2}^{2}\right)^{1 / 2} .
$$

Here, $E_{2 \mathrm{~d}}(k) /(2 \pi k)$ is the spectral energy density that is constant within a ring with circumference $2 \pi k$ for $2 \mathrm{D}$ isotropic turbulence, and the prefactor is $2 / \pi=2 \times 2 /(2 \pi)$ because $E_{1 \mathrm{~d}}\left(k_{1}\right)$ is defined for positive $k_{1}$, and the integral is taken over positive $k_{2}$ wavenumbers only (E. Lindborg 2019, personal communication). Equation (A10) can be evaluated numerically. Computed 2D spectra differ from the $1 \mathrm{D}$ spectra by factors between 0.7 and 3 depending of the slope. The factor is largest for spectra with positive slope.

\section{REFERENCES}

Bacmeister, J. T., S. D. Eckermann, P. A. Newman, L. Lait, K. R. Chan, M. Loewenstein, M. H. Proffitt, and B. L. Gary, 1996: Stratospheric horizontal wavenumber spectra of winds, potential temperature, and atmospheric tracers observed by high-altitude aircraft.J. Geophys. Res., 101, 9441-9470, https:// doi.org/10.1029/95JD03835.

Barahona, D., A. Molod, and H. Kalesse, 2017: Direct estimation of the global distribution of vertical velocity within cirrus clouds. Sci. Rep., 7, 6840, https://doi.org/10.1038/s41598-017-07038-6.

Bierdel, L., C. Snyder, S.-H. Park, and W. C. Skamarock, 2016: Accuracy of rotational and divergent kinetic energy spectra diagnosed from flight-track winds. J. Atmos. Sci., 73, 3273-3286, https:// doi.org/10.1175/JAS-D-16-0040.1.

Birner, T., A. Dörnbrack, and U. Schumann, 2002: How sharp is the tropopause at midlatitudes? Geophys. Res. Lett., 29, 1700, https://doi.org/10.1029/2002GL015142.

Blažica, V., N. Žagar, B. Strajnar, and J. Cedilnik, 2013: Rotational and divergent kinetic energy in the mesoscale model ALADIN. Tellus, 65A, 18918, https://doi.org/10.3402/tellusa.v65i0.18918. 
Bony, S., and B. Stevens, 2019: Measuring area-averaged vertical motions with dropsondes. J. Atmos. Sci., 76, 767-783, https:// doi.org/10.1175/JAS-D-18-0141.1.

Bühler, O., M. Kuang, and E. G. Tabak, 2017: Anisotropic Helmholtz and wave-vortex decomposition of one-dimensional spectra. J. Fluid Mech., 815, 361-387, https://doi.org/10.1017/jfm.2017.57.

Burgess, B. H., A. R. Erler, and T. G. Shephard, 2013: The troposphereto-stratosphere transition in kinetic energy spectra and nonlinear spectral fluxes as seen in ECMWF analyses. J. Atmos. Sci., 70, 669-687, https://doi.org/10.1175/JAS-D-12-0129.1.

Callies, J., O. Bühler, and R. Ferrari, 2016: The dynamics of mesoscale winds in the upper troposphere and lower stratosphere. J. Atmos. Sci., 73, 4853-4872, https://doi.org/10.1175/JAS-D-16-0108.1.

Charney, J. G., 1971: Geostrophic turbulence. J. Atmos. Sci., 28 , 1087-1095, https://doi.org/10.1175/1520-0469(1971)028<1087: $\mathrm{GT}>2.0 . \mathrm{CO} ; 2$.

Chen, T.-C., and J. J. Tribbia, 1981: Kinetic energy spectra of divergent wind in the atmosphere. Tellus, 33, 102-104, https:// doi.org/10.3402/tellusa.v33i1.10698.

Cho, J. Y. N., R. E. Newell, and J. D. Barrick, 1999: Horizontal wavenumber spectra of winds, temperature, and trace gases during the Pacific Exploratory Missions: 2. Gravity waves, quasi-twodimensional turbulence, and vortical modes. J. Geophys. Res., 104, 16297-16308, https://doi.org/10.1029/1999JD900068.

Cooper, W. A., S. M. Spuler, M. Spowart, D. H. Lenschow, and R. B. Friesen, 2014: Calibrating airborne measurements of airspeed, pressure and temperature using a Doppler laser airmotion sensor. Atmos. Meas. Tech., 7, 3215-3231, https://doi.org/ 10.5194/amt-7-3215-2014.

Craig, G. C., and T. Selz, 2018: Mesoscale dynamical regimes in the midlatitudes. Geophys. Res. Lett., 45, 410-417, https://doi.org/ 10.1002/2017GL076174.

Dewan, E. M., 1979: Stratospheric wave spectra resembling turbulence. Science, 204, 832-835, https://doi.org/10.1126/ science.204.4395.832.

Egger, J., and K.-P. Hoinka, 2019: Hydrostatic vertical velocity and incompressibility in the Northern Hemisphere. Quart. J. Roy. Meteor. Soc., 145, 563-574, https://doi.org/10.1002/qj.3452.

Frehlich, R. G., and R. D. Sharman, 2008: The use of structure functions and spectra from numerical model output to determine effective model resolution. Mon. Wea. Rev., 136 1537-1553, https://doi.org/10.1175/2007MWR2250.1.

— and - 2010: Equivalence of velocity statistics at constant pressure or constant altitude. Geophys. Res. Lett., 37, L08801, https://doi.org/10.1029/2010GL042912.

Fritts, D. C., and M. J. Alexander, 2003: Gravity wave dynamics and effects in the middle atmosphere. Rev. Geophys., 41, 1003, https://doi.org/10.1029/2001RG000106.

— , and Coauthors, 2016: The Deep Propagating Gravity Wave Experiment (DEEPWAVE): An airborne and ground-based exploration of gravity wave propagation and effects from their sources throughout the lower and middle atmosphere. Bull. Amer. Meteor. Soc., 97, 425-453, https://doi.org/10.1175/BAMS-D-14-00269.1.

Gage, K. S., and G. D. Nastrom, 1986: Theoretical interpretation of atmospheric wavenumber spectra of wind and temperature observed by commercial aircraft during GASP. J. Atmos. Sci., 43, 729-740, https://doi.org/10.1175/1520-0469(1986)043<0729: TIOAWS $>2.0 . \mathrm{CO} ; 2$.

Gao, X., and J. W. Meriwether, 1998: Mesoscale spectral analysis of in situ horizontal and vertical wind measurements at $6 \mathrm{~km}$. J. Geophys. Res., 103, 6397-6404, https://doi.org/10.1029/97JD03074.

Geller, M. A., and J. Gong, 2010: Gravity wave kinetic, potential, and vertical fluctuation energies as indicators of different frequency gravity waves. J. Geophys. Res., 115, D11111, https:// doi.org/10.1029/2009JD012266.

Giez, A., C. Mallaun, M. Zöger, A. Dörnbrack, and U. Schumann, 2017: Static pressure from aircraft trailing-cone measurements and numerical weather-prediction analysis. J. Aircr., 54, 1728-1737, https://doi.org/10.2514/1.C034084.

Gill, A. E., 1982: Atmosphere-Ocean Dynamics. Academic Press, $662 \mathrm{pp}$.

Gisinger, S., and Coauthors, 2017: Atmospheric conditions during the Deep Propagating Gravity Wave Experiment (DEEPWAVE). Mon. Wea. Rev., 145, 4249-4275, https://doi.org/10.1175/ MWR-D-16-0435.1.

Gultepe, I., and D. O. C. Starr, 1995: Dynamical structure and turbulence in cirrus clouds: Aircraft observations during FIRE. J. Atmos. Sci., 52, 4159-4182, https://doi.org/10.1175/ 1520-0469(1995)052<4159:DSATIC>2.0.CO;2.

Hamilton, K., Y. O. Takahashi, and W. Ohfuchi, 2008: Mesoscale spectrum of atmospheric motions investigated in a very fine resolution global general circulation model. J. Geophys. Res., 113, D18110, https://doi.org/10.1029/2008JD009785.

Heinze, R., and Coauthors, 2017: Large-eddy simulations over Germany using ICON: A comprehensive evaluation. Quart. J. Roy. Meteor. Soc., 143, 69-100, https://doi.org/10.1002/qj.2947.

Hinze, J. O., 1959: Turbulence. McGraw-Hill, 586 pp.

Holton, J., 2012: An Introduction to Dynamic Meteorology. Academic Press, 529 pp.

Jasperson, W. H., G. D. Nastrom, and D. C. Fritts, 1990: Further study of terrain effects on the mesoscale spectrum of atmospheric motions. J. Atmos. Sci., 47, 979-987, https://doi.org/10.1175/ 1520-0469(1990)047<0979:FSOTEO > 2.0.CO;2.

Kang, S.-L., and D. H. Lenschow, 2014: Temporal evolution of lowlevel winds induced by two-dimensional mesoscale surface heat-flux heterogeneity. Bound-Layer Meteor., 151, 501-529, https://doi.org/10.1007/s10546-014-9912-8.

Kärcher, B., E. J. Jensen, and U. Lohmann, 2019: The impact of mesoscale gravity waves on homogeneous ice nucleation in cirrus clouds. Geophys. Res. Lett., 46, 5556-5565, https://doi.org/ 10.1029/2019GL082437.

Kolmogorov, A., 1941: The local structure of turbulence in incompressible viscous fluids for very large Reynolds number. Dokl. Akad. Nauk SSSR, 30, 301-305.

Koshyk, J. N., and K. Hamilton, 2001: The horizontal kinetic energy spectrum and spectral budget simulated by a highresolution troposphere-stratosphere-mesosphere GCM. J. Atmos. Sci., 58, 329-348, https://doi.org/10.1175/1520-0469(2001) 058<0329:THKESA $>2.0 . \mathrm{CO} ; 2$.

Lenschow, D. H., 1986: Aircraft measurements in the boundary layer. Probing the Atmospheric Boundary Layer, D. H. Lenschow, Ed., Amer. Meteor. Soc., 39-55.

Li, Q., and E. Lindborg, 2018: Weakly or strongly nonlinear mesoscale dynamics close to the tropopause? J. Atmos. Sci., 75, 1215-1229, https://doi.org/10.1175/JAS-D-17-0063.1.

Li, Q., M. Rapp, G. Stober, and R. Latteck, 2018: High-resolution vertical velocities and their power spectrum observed with the MAARSY radar-Part 1: Frequency spectrum. Ann. Geophys., 36, 577-586, https://doi.org/10.5194/angeo-36-577-2018.

Lilly, D. K., 1967: The representation of small-scale turbulence in numerical simulation experiments. IBM Scientific Computing Symp. on Environmental Science, Yorktown Heights, NY, IBM, 195-210.

1978: A severe downslope windstorm and aircraft turbulence event induced by a mountain wave. J. Atmos. Sci. 35, 59-77, https:// doi.org/10.1175/1520-0469(1978)035<0059:ASDWAA>2.0.CO;2. 
_ 1983: Stratified turbulence and the mesoscale variability of the atmosphere. J. Atmos. Sci., 40, 749-761, https://doi.org/ 10.1175/1520-0469(1983)040<0749:STATMV>2.0.CO;2.

Lindborg, E., 1999: Can the atmospheric energy spectrum be explained by two-dimensional turbulence? J. Fluid Mech., 388, 259-288, https://doi.org/10.1017/S0022112099004851.

, 2007: Horizontal wavenumber spectra of vertical vorticity and horizontal divergence in the upper troposphere and lower stratosphere. J. Atmos. Sci., 64, 1017-1025, https://doi.org/ 10.1175/JAS3864.1.

2009: Two comments on the surface quasigeostrophic model for the atmospheric energy spectrum. J. Atmos. Sci., 66, 1069-1072, https://doi.org/10.1175/2008JAS2972.1.

, 2015: A Helmholtz decomposition of structure functions and spectra calculated from aircraft data. J. Fluid Mech., 762, R4, https://doi.org/10.1017/jfm.2014.685.

Lorenz, E. N., 1960: Energy and numerical weather prediction. Tellus, 12, 364-373, https://doi.org/10.1111/j.2153-3490. 1960.tb01323.x.

__ 1969: The predictability of a flow which possesses many scales of motion. Tellus, 21, 289-307, https://doi.org/10.1111/ j.2153-3490.1969.tb00444.x.

Lynch, P., 2003: Margules's tendency equation and Richardson's forecast. Weather, 58, 186-193, https://doi.org/10.1256/wea.146.02.

Malardel, S., and N. P. Wedi, 2016: How does subgrid-scale parametrisation influence nonlinear spectral energy fluxes in global NWP models? J. Geophys. Res. Atmos., 121, 5395-5410, https://doi.org/10.1002/2015JD023970.

Mallaun, C., A. Giez, and R. Baumann, 2015: Calibration of 3-D wind measurements on a single engine research aircraft. Atmos. Meas. Tech., 8, 3177-3196, https://doi.org/10.5194/amt-8-3177-2015.

Menchaca, M. Q., and D. R. Durran, 2019: The influence of gravity waves on the slope of the kinetic energy spectrum in simulations of idealized midlatitude cyclones. J. Atmos. Sci., 76, 2103-2122, https://doi.org/10.1175/JAS-D-18-0329.1.

Nastrom, G. D., and K. S. Gage, 1985: A climatology for atmospheric wavenumber spectra of wind and temperature observed by commercial aircraft. J. Atmos. Sci., 42, 950-960, https://doi.org/10.1175/ 1520-0469(1985)042<0950:ACOAWS>2.0.CO;2.

—_ and D. C. Fritts, 1992: Sources of mesoscale variablity of gravity waves. Part I: Topographic excitation. J. Atmos. Sci., 49, 101-110, https://doi.org/10.1175/1520-0469(1992)049<0101: SOMVOG $>2.0 . \mathrm{CO} ; 2$.

$\longrightarrow,-$, and K. S. Gage, 1987: An investigation of terrain effects on the mesoscale spectrum of atmospheric motions. J. Atmos. Sci., 44, 3087-3096, https://doi.org/10.1175/1520-0469(1987) 044<3087:AIOTEO > 2.0.CO;2.

__, T. E. Van Zandt, and J. M. Warnock, 1997: Vertical wavenumber spectra of wind and temperature from highresolution balloon soundings over Illinois. J. Geophys. Res., 102, 6685-6701, https://doi.org/10.1029/96JD03784.

Nelson, R. C., 1998: Flight Stability and Automatic Control. McGraw-Hill, 441 pp.

Peltier, L. J., J. C. Wyngaard, S. Khanna, and J. O. Brasseur, 1996: Spectra in the unstable surface layer. J. Atmos. Sci., 53, 49-61, https://doi.org/10.1175/1520-0469(1996)053<0049: SITUSL $>2.0 . C O ; 2$.

Podglajen, A., A. Hertzog, R. Plougonven, and B. Legras, 2016: Lagrangian temperature and vertical velocity fluctuations due to gravity waves in the lower stratosphere. Geophys. Res. Lett., 43, 3543-3553, https://doi.org/10.1002/2016GL068148.

Riley, J. J., and E. Lindborg, 2008: Stratified turbulence: A possible interpretation of some geophysical turbulence measurements.
J. Atmos. Sci., 65, 2416-2424, https://doi.org/10.1175/ 2007JAS2455.1.

Schäfler, A., and Coauthors, 2018: The North Atlantic Waveguide and Downstream Impact Experiment. Bull. Amer. Meteor. Soc., 99, 1607-1637, https://doi.org/10.1175/BAMS-D-17-0003.1.

Schumann, U., P. Konopka, R. Baumann, R. Busen, T. Gerz, H. Schlager, P. Schulte, and H. Volkert, 1995: Estimate of diffusion parameters of aircraft exhaust plumes near the tropopause from nitric oxide and turbulence measurements. J. Geophys. Res., 100, 14 147-14 162, https://doi.org/10.1029/95JD01277.

Selz, T., L. Bierdel, and G. C. Craig, 2019: Estimation of the variability of mesoscale energy spectra with three years of COSMO-DE analyses. J. Atmos. Sci., 76, 627-637, https:// doi.org/10.1175/JAS-D-18-0155.1.

Sharman, R. D., S. B. Trier, T. P. Lane, and J. D. Doyle, 2012: Sources and dynamics of turbulence in the upper troposphere and lower stratosphere: A review. Geophys. Res. Lett., 39, L12803, https://doi.org/10.1029/2012GL051996.

_ , L. B. Cornman, G. Meymaris, J. Pearson, and T. Farrar, 2014: Description and derived climatologies of automated in situ eddydissipation-rate reports of atmospheric turbulence. J. Appl. Meteor. Climatol., 53, 1416-1432, https://doi.org/10.1175/JAMC-D-13-0329.1.

Skamarock, W. C., S.-H. Park, J. B. Klemp, and C. Snyder, 2014: Atmospheric kinetic energy spectra from global high-resolution nonhydrostatic simulations. J. Atmos. Sci. 71, 4369-4381, https:// doi.org/10.1175/JAS-D-14-0114.1.

_ C. Snyder, J. B. Klemp, and S.-H. Park, 2019: Vertical resolution requirements in atmospheric simulation. Mon. Wea. Rev., 147, 2641-2656, https://doi.org/10.1175/MWR-D-19-0043.1.

Smith, R. B., and C. G. Kruse, 2017: Broad-spectrum mountain waves. J. Atmos. Sci., 74, 1381-1402, https://doi.org/10.1175/ JAS-D-16-0297.1.

— , and Coauthors, 2016: Stratospheric gravity wave fluxes and scales during DEEPWAVE. J. Atmos. Sci., 73, 2851-2869, https:// doi.org/10.1175/JAS-D-15-0324.1.

Tennekes, H., and J. L. Lumley, 1972: A First Course in Turbulence. MIT Press, $300 \mathrm{pp}$.

Tong, C., and K. X. Nguyen, 2015: Multipoint Monin-Obukhov similarity and its application to turbulence spectra in the convective atmospheric surface layer. J. Atmos. Sci., 72, 4337-4348, https://doi.org/10.1175/JAS-D-15-0134.1.

Waite, M. L., and C. Snyder, 2009: The mesoscale kinetic energy spectrum of a baroclinic life cycle. J. Atmos. Sci., 66, 883-901, https://doi.org/10.1175/2008JAS2829.1.

Wippermann, F., 1957: Die Transformation potentieller und innerer Energie in die Energie rotorloser und diejenige divergenzfreier Bewegungen (Transformation of potential and internal energy into energy of irrotational motions and into energy of nondivergent motions). Beitr. Phys. Atmos., 29, 269-275.

Witschas, B., S. Rahm, A. Dörnbrack, J. Wagner, and M. Rapp, 2017: Airborne wind lidar measurements of vertical and horizontal winds for the investigation of orographically induced gravity waves. J. Atmos. Oceanic Technol., 34, 1371-1386, https://doi.org/10.1175/JTECH-D-17-0021.1.

Zentek, R., G. Heinemann, and E. Sachs, 2016: Climatology of wind, kinetic energy, and temperature spectra using a highresolution climate model for mid-Europe. J. Climate, 29, 963-974, https://doi.org/10.1175/JCLI-D-15-0540.1.

Zhang, F., J. Wei, M. Zhang, K. P. Bowman, L. L. Pan, E. Atlas, and S. C. Wofsy, 2015: Aircraft measurements of gravity waves in the upper troposphere and lower stratosphere during the START08 field experiment. Atmos. Chem. Phys., 15, 7667-7684, https://doi.org/10.5194/acp-15-7667-2015. 